\title{
Simulation studies of modulator for coherent electron cooling
}

\author{
Jun Ma and Xingyu Wang \\ Stony Brook University, Stony Brook, New York 11794, USA \\ Gang Wang and Kwangmin Yu \\ Brookhaven National Laboratory, Upton, New York 11973, USA \\ Roman Samulyak* and Vladimir Litvinenko \\ Stony Brook University, Stony Brook, New York 11794, USA \\ and Brookhaven National Laboratory, Upton, New York 11973, USA
}

(Received 1 August 2017; published 12 November 2018)

\begin{abstract}
Interaction of hadrons with electron beam in a modulator is an important part of coherent electron cooling $(\mathrm{CeC})$, a novel cooling method for hadron beams. Being an untested technique, the $\mathrm{CeC}$ is undergoing a proof-of-principle test at Brookhaven National Laboratory (BNL). Simulation of this process for a realistic electron beam propagating through a realistic quadrupole beamline constitutes a very challenging problem. We successfully used the code SPACE for these simulations and obtained accurate dependences of the modulation process on the position and velocity of ions. We obtained good numerical convergence of simulations and performed verification tests using theoretical predications available for a uniform infinite plasma with $\kappa-2$ velocity distribution. In this paper, we describe simulation methods and results, and report our findings for the $\mathrm{CeC}$ modulator in the $\mathrm{BNL}$ experiment.
\end{abstract}

DOI: 10.1103/PhysRevAccelBeams.21.111001

\section{INTRODUCTION}

Cooling of high-energy hadron beams is among major challenges in modern accelerator physics. Coherent electron cooling $(\mathrm{CeC})$ [1-3] is a novel technique developed for rapid cooling of high-energy, high-intensity hadron beams. $\mathrm{CeC}$ consists of three main components: a modulator, where each ion imprints a density wake on the electron distribution, a free electron laser (FEL) as an amplifier, where the density wakes are amplified, and a kicker, where the amplified wakes interacts with ions, resulting in dynamical friction for the ion that leads to cooling of ion beams. Figure 1 shows a schematic of $\mathrm{CeC}$. In advanced coherent electron cooling $(\mathrm{ACeC})$ [4,5], the free electron laser is replaced by a three-pole wiggler.

Despite significant differences in their amplifiers, all proposed $\mathrm{CeC}$ systems share the same mechanism for the modulation process: Coulomb interactions between the ions and the surrounding electrons [6]. The relative modulation of the density of electrons due to their interaction with ions is orders of magnitudes smaller than unity and, consequently, it

\footnotetext{
*roman.samulyak@stonybrook.edu
}

Published by the American Physical Society under the terms of the Creative Commons Attribution 4.0 International license. Further distribution of this work must maintain attribution to the author(s) and the published article's title, journal citation, and DOI. is viable to treat each ion individually and use the superposition principle to obtain the net responses of electrons to all ions in the beam. There is an analytic solution to the modulator problem involving an ion moving in a cloud of electrons with uniform spatial distribution [7]. For a system with spatially nonuniform electrons, numerical approaches are employed that either solve the Vlasov equation [8] directly or use macroparticle simulations [9]. One of the difficulties in a macroparticle simulation originates from the fact that the signal due to the modulation is too weak compared to the shot noise resulting from the discreteness of macroparticles. In the approach adopted by G. Bell et al. [9], the difficulties are overcome by splitting the electrons into two groups, the background electrons and the $\delta f$ electrons that are involved in the modulation process. Since only the $\delta f$ electrons contain information about the modulation, the signal-to-noise ratio is significantly enhanced. In this work, we simulate the perturbation in the electron beam density via highly resolved simulations and follow a different approach to extract the modulation signal from the shot noise. We perform two simulations with identical initial distributions of electrons, one in the presence of an ion and the other propagating electrons without the ion. We take difference in final electron distributions of the two simulations to obtain the influence of the ion. Details of this method are described in Sec. II C.

In our work, we use the code SPACE [10], a 3D electromagnetic particle-in-cell (PIC) code, which contains fully 


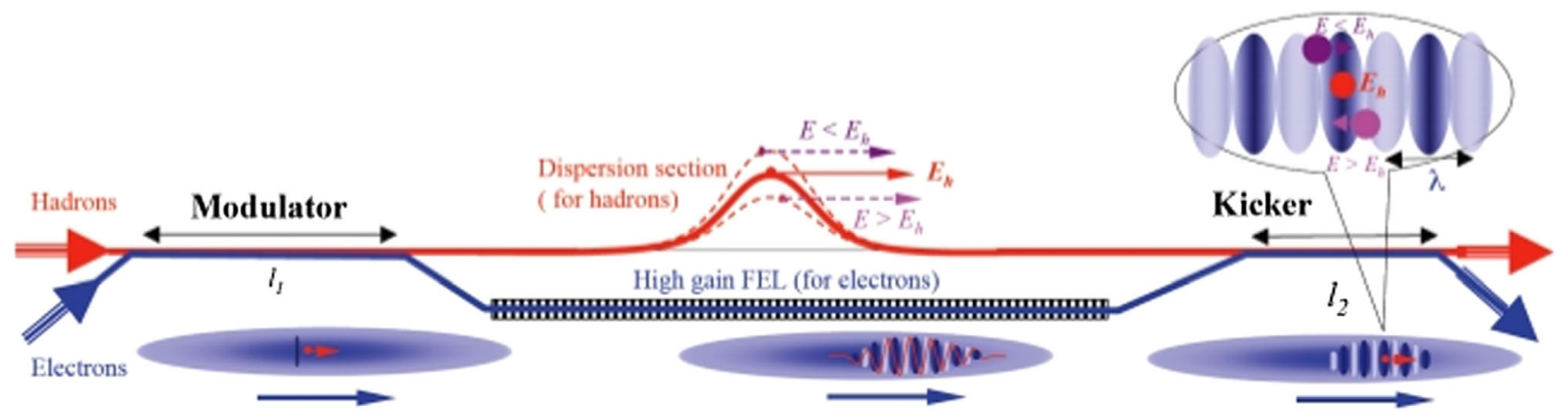

FIG. 1. Schematic of coherent electron cooling concept.

electromagnetic solvers for relativistic particles and electrostatic solvers for Poisson-Vlasov equation. The electrostatic solvers implement the traditional PIC method as well as a new method of Adaptive Particle-in-Cloud [11]. The code has been recently used for the study of plasma dynamics in a dense gas filled rf cavities [12], designed for ionization cooling experiments, simulation of laser-induced wakefields in plasma, and processes in a particle beam-induced plasma relevant to the mitigation of beam-beam effects [13].

\section{METHODS, TOOLS, AND PROBLEM SETUP}

\section{A. Code SPACE}

SPACE is a parallel, relativistic, 3D electromagnetic particle-in-cell code [10] developed for the simulation of relativistic particle beams, beam-plasma interaction, and plasma chemistry. It also contains modules for solving equations in the electrostatic approximation. This code module was used in the comoving frame of the ions and electrons, where the interaction is essentially electrostatic. The electrostatic module of SPACE employs two different approaches. The first one is the traditional PIC method for the Poisson-Vlasov equation, based on a uniform Cartesian mesh, a linear charge deposition scheme, and fast fourier transform (FFT) solvers.
In additions to the PIC solver, the code includes an implementation of the new highly adaptive particle-in-cloud (AP-Cloud) method [11] that replaces the traditional PIC mesh with an adaptively chosen set of computational particles on an octree data structure and uses the weighed least squares method for approximation of differential and integral operators. The AP-Cloud code is especially beneficial if the distribution of particles is nonuniform and / or the computational domain is geometrically irregular or boundary conditions are of mixed type, such as open boundary condition in the transverse directions and periodic in the longitudinal direction. We have compared results of PIC and AP-Cloud simulations of modulator-related problems described below. Both approaches have passed various verification tests. While PIC and AP-Cloud results were generally in good agreement, we found that the AP-Cloud methods produced higher accuracy for Gaussian beams in computational domains with mixed boundary conditions. In addition, AP-Cloud automatically selects an optimal local numerical resolution, as described below. Therefore, all simulations reported in this paper were obtained using the AP-Cloud method.

\section{B. Parameters of $\mathrm{CeC}$ modulator}

The main focus of our studies is the ongoing proof of the $\mathrm{CeC}$ principle experiment in the Relativistic Heavy Ion

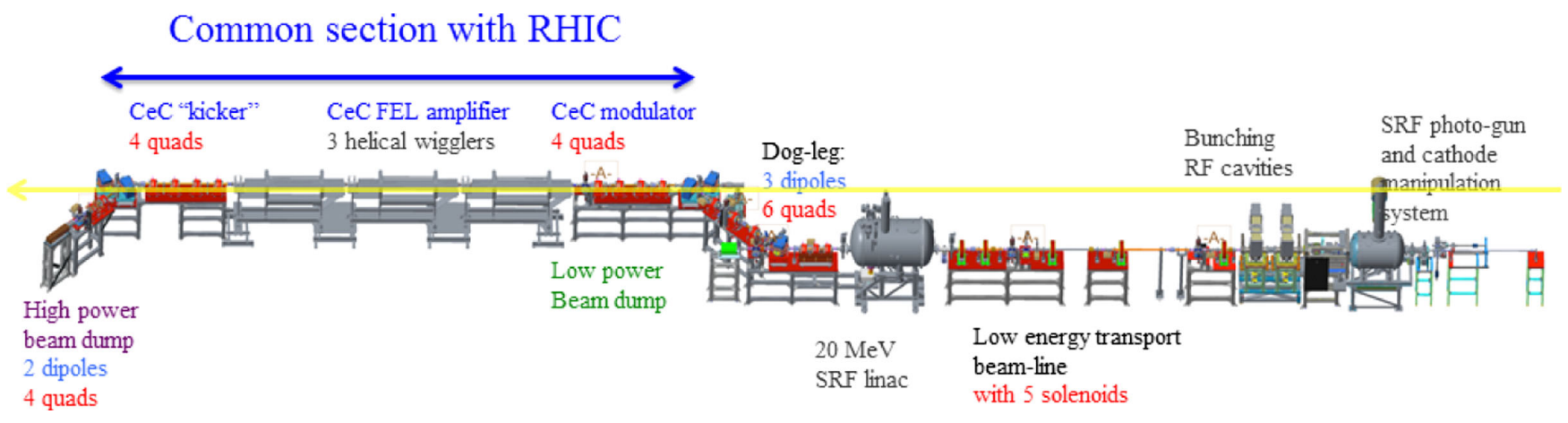

FIG. 2. The CeC system at BNL. It includes the following equipment: $112 \mathrm{MHz}$ SRF gun; $500 \mathrm{MHz}$ RF buncher/pre-accelerator; $20 \mathrm{MeV} 704 \mathrm{MHz}$ SRF module; electron beam transport magnets (dipoles, quadrupoles, solenoids, trims); helical FEL wigglers; beam dump; and RHIC DX-, D0-, and triplet-magnets. The electron beam is merged with the ion beam using an achromatic dog-leg, cools the ions in the $\mathrm{CeC}$, and then is discarded into a beam dump. 
TABLE I. Parameters of ion beam.

\begin{tabular}{lc}
\hline \hline Parameter & Ion beam, $\mathrm{Au}^{+79}$ \\
\hline Beam energy & $\gamma=42.9$ \\
Bunch intensity & $1 \times 10^{9}$ \\
Bunch length & $60 \mathrm{~cm}$ (r.m.s.) \\
RMS emittance (normalized) & $2 \pi \mathrm{mm} \mathrm{mrad}$ \\
\hline \hline
\end{tabular}

TABLE II. Parameters of electron beams.

\begin{tabular}{lc}
\hline \hline Parameter & Electron beam \\
\hline Beam energy & $\gamma=42.9$ \\
Peak current & $100 \mathrm{~A}$ \\
Bunch intensity & $1 \mathrm{nC}$ \\
Bunch length & $10 \mathrm{ps}(\mathrm{full})$ \\
RMS emittance (normalized) & $5 \pi \mathrm{mm} \mathrm{mrad}$ \\
Relative RMS energy spread & $1 \mathrm{e}-3$ \\
Beta function at modulator & $4 \mathrm{~m}$ \\
Plasma frequency (lab frame) & $1.5 \mathrm{e}+8 \mathrm{rad} / \mathrm{s}$ \\
Transverse Debye length (lab frame) & $3.4 \mathrm{e}-4 \mathrm{~m}$ \\
Longitudinal Debye length (lab frame) & $1.1 \mathrm{e}-6 \mathrm{~m}$ \\
\hline \hline
\end{tabular}

Collider (RHIC) at the Brookhaven National Laboratory (BNL). The experiment is located at IR2 of the RHIC and the layout of the experiment is shown in Fig. 2. The electrons are generated from the $112 \mathrm{MHz}$ SRF gun with 1-3 nC of charge per bunch. After being bunched with two $500 \mathrm{MHz}$ normal conducing rf cavities, the electrons are accelerated to the energy of $15-20 \mathrm{MeV}$ in the $704 \mathrm{MHz}$ SRF linac. Full energy electron beam propagates through the $\mathrm{CeC}$ section common with RHIC, where it interacts with ions circulating in RHIC's Yellow ring. At the end of the $\mathrm{CeC}$ section, the electrons are bent into the beam dump. As shown in Fig. 2, the modulator section of the $\mathrm{CeC}$ system consists of four quadrupoles which are used to control traverse dynamics of electron beam as well as to match it to the downstream FEL amplifier. The parameters of the electron and ion beams are listed in Tables I and II.

\section{Extracting modulation signal}

The purpose of modulator simulations is to quantify the density and velocity distributions of electrons after their interaction with a gold ion. The detection of the modulation process is a very difficult task due to the presence of strong shot noise that greatly reduces the signal-to-noise ratio. The generation of initial positions and velocities for computational particles representing electrons is performed using random number generators and prescribed probability density functions. Nonuniformities in space and thermal motion due to the random generation contribute to the shot noise and are orders of magnitude larger compared to physical redistributions due to the interaction of particles.

We have tested two methods for the reduction of the shot noise. The first one improves the statistics by reducing the representing number of computational particles. The representing number determines how many real particles are represented by a computational particle, and its typical value in the present simulations is 1 (single electrons are resolved). However, even with the representing number of one, the shot noise is still significantly stronger than the modulation signal due to the interaction of electrons with an ion. To reduce the shot noise, we performed simulations with electron representing numbers $\ll 1$. This method, while being computationally very intensive, is still insufficient: the use of representing number of 0.05 resulted in the signal-to-background noise ratio of the order of one percent.

The second method proved to be much more effective without causing a significant increase of the computational cost. For each specific modulation problem, we perform two simulations that use identical initial distributions of electrons. One simulation operates only with the electron beam while in the other simulation, the electron beam copropagates with an ion. With the assumption that the Coulomb force from the ion only slightly changes the trajectories of the cooling electrons over the course of $\mathrm{CeC}$ modulation, the influences of the ion can be obtained by taking the difference in the final electron distributions of the two simulations. This method effectively eliminates the shot noise caused by the randomness of the background and gives a clear modulation signal. A similar approach has also been successfully applied to simulate the FEL amplification process in the presence of shot noise [14].

\section{SIMULATION RESULTS}

The modulation process strongly depends on the temperature of electrons. Since the parallel scalability of our code makes it possible to resolve all physical electrons in relevant spatial domains defined by the Debye length, the thermal velocity distribution of electrons is easy to implement. For the verification tests that involved comparison with analytical solutions, thermal velocity distributions of spatially uniform electron beams were modeled consistently with the assumptions of the theory, namely using a " $\kappa-2$ " probability density function (1)

$$
\begin{aligned}
f_{0}(\vec{v})= & \frac{n_{0}}{\pi^{2} \sigma_{v_{x}} \sigma_{v_{y}} \sigma_{v_{z}}} \\
& \times\left(1+\frac{\left(v_{x}+v_{0, x}\right)^{2}}{\sigma_{v_{x}}^{2}}+\frac{\left(v_{y}+v_{0, y}\right)^{2}}{\sigma_{v_{y}}^{2}}+\frac{\left(v_{z}+v_{0, z}\right)^{2}}{\sigma_{v_{z}}^{2}}\right)^{-2},
\end{aligned}
$$

where $\sigma_{v_{x}}, \sigma_{v_{y}}$, and $\sigma_{v_{z}}$ are characteristic velocities in 3 -dimensional Cartesian coordinates and $\vec{v}_{0}$ is the velocity of the ion. In this case, spatial density of electrons is assumed to be uniform. Reductions of Eq. (1) to 1D and 2D are given in [15]. 


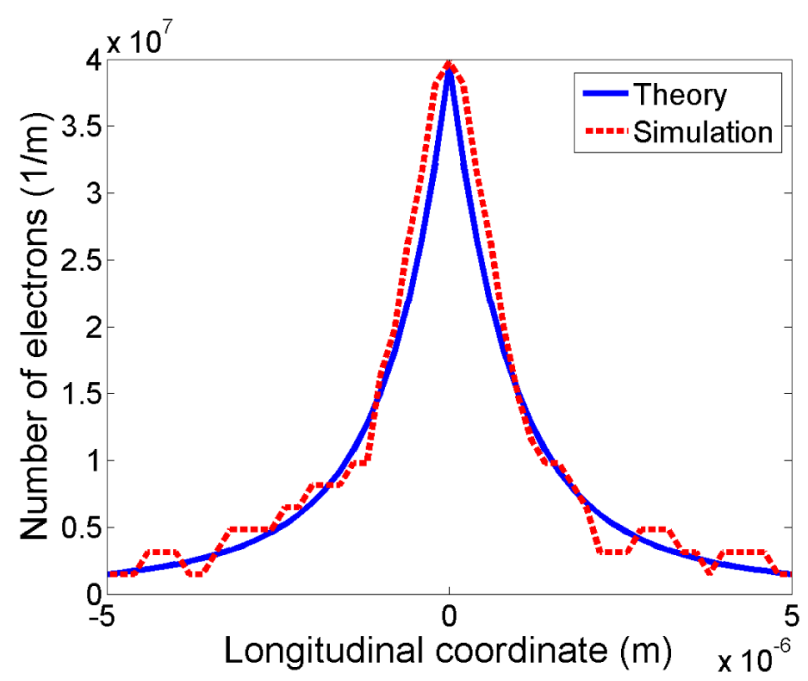

(a) Longitudinal density

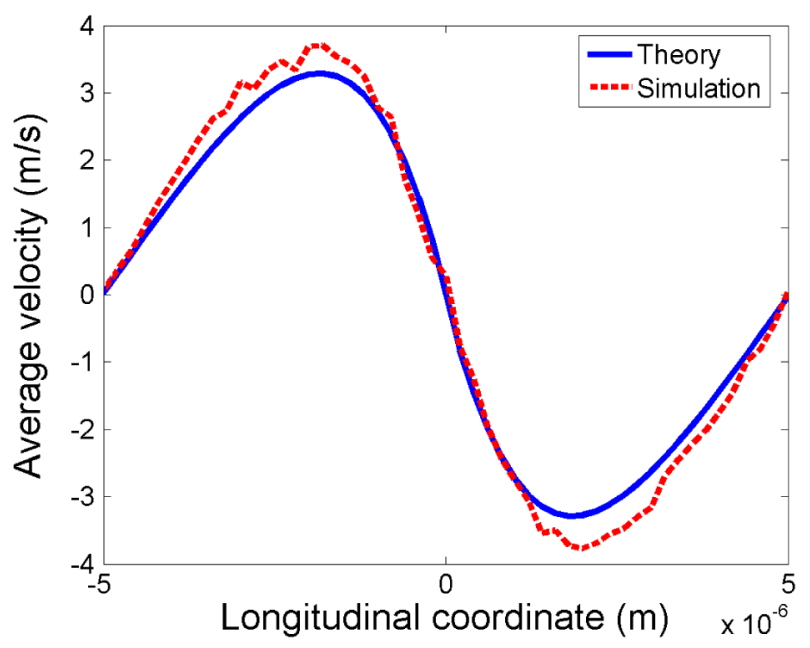

(b) Longitudinal velocity

FIG. 3. Comparison of theory and numerical simulations of density (a) and velocity (b) modulation by stationary ion with respect to uniform electron cloud.

For the simulation of the modulation processes in Gaussian electron beams with linear and quadruple focusing fields, we used realistic thermal velocity distributions described below.

The modulation process is studied numerically by placing a single ion in an electron beam. This approximation, justified by negligibly small ion-ion interactions on length scales relevant to the modulation process, significantly reduces the computation time. As two beams copropagate with the same averaged speed, simulations are performed in a relativistic frame moving with the velocity of the ion and the electron beams. In the moving frame, the thermal velocity distribution parameters are chosen as $\sigma_{v_{x}}=$ $\sigma_{v_{y}}=2 \times 10^{6} \mathrm{~m} / \mathrm{s}$ and $\sigma_{v_{z}}=3 \times 10^{5} \mathrm{~m} / \mathrm{s}$, consistent with experimental predictions.

\section{A. Verification studies}

For our verification tests, we used a single ion in the center of a computational domain of 5 Debye length in each dimension with periodic boundary conditions in all directions. The domain is uniformly filled with electrons that satisfy the $\kappa-2$ thermal velocity distribution (1). We then compared our results with a known analytical solution [15].

One advantage of the AP-Cloud method over PIC [11] is its ability to select automatically an optimal numerical resolution for the given density of physical particles. This is accomplished by selecting an optimal number of computational particles that play similar role as the PIC grid cells. The criterion for the optimal numerical resolution is the balance of two main errors in the discretization of the Poisson equation for the electric potential. The first one is associated with the evaluation of the charge density in the right-hand side and has the nature of the Monte-Carlo integration noise. The other error is associated with the truncation error of the numerical approximation of the differential operator (Laplacian). As a result, the resolution is always dynamically and automatically adjusted depending on the density of physical macroparticles. We have checked that numerical results have reached numerical convergence: they remain essentially the same with the increase of the number of macroparticles.

Simulations performed using a uniform distribution of electrons, periodic boundary conditions, and the $\kappa-2$ thermal velocity distribution can be directly compared with theoretical predictions. Theoretical value for the density modulation, obtained in [15], is

$$
\tilde{n}_{1}(x, t)=\frac{Z_{i} \omega_{p}}{\pi \sigma_{v}} \int_{0}^{t} \frac{\psi \sin \left(\omega_{p} \psi\right) d \psi}{\psi^{2}+\frac{\left(x+v_{0} \psi\right)^{2}}{\beta^{2}}},
$$

where $\tilde{n}_{1}(x, t)$ is the shielding response of the electrons to the ion, $Z_{i}$ is the charge number of the ion $\left(Z_{i}=79\right.$ for a fully stripped gold ion), $\omega_{p}$ is plasma frequency, and $\sigma_{v}$ is the characteristic velocity spread of electrons. In the limiting case of $t \rightarrow \infty$, Eq. (2) gives the steady-state solution [15]

$$
\tilde{n}_{1}(x)=\frac{Z_{i} \omega_{p}}{2 \sigma_{v}} \exp \left(-\frac{\omega_{p}|x|}{\beta}\right) .
$$

The corresponding theoretical values for the velocity modulation are derived from the analytic solution for the energy modulation [16].

For a stationary ion in the moving frame, simulation results are in very good agreement with theoretical values of the density modulation [Fig. 3(a)] and the velocity modulation [Fig. 3(b)]. 


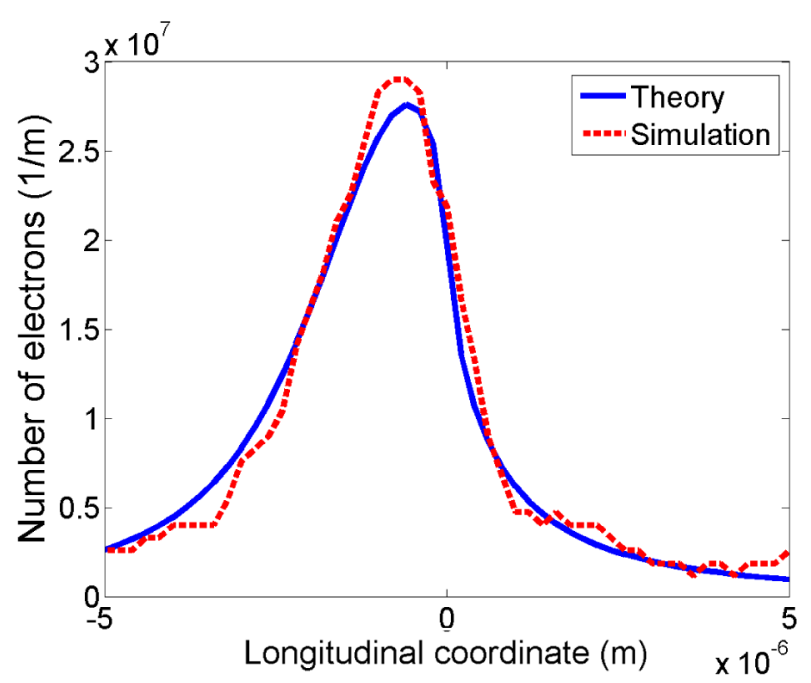

(a) Longitudinal density

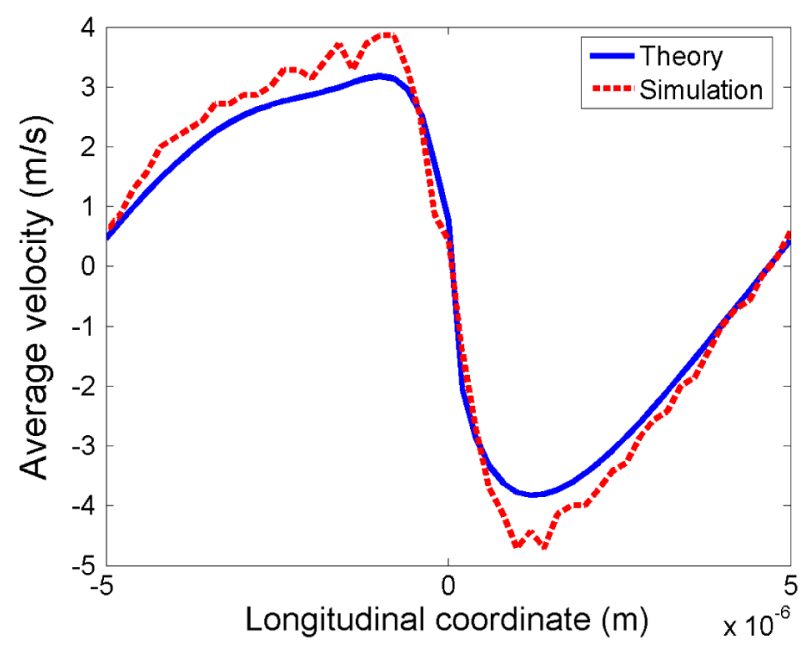

(b) Longitudinal velocity

FIG. 4. Comparison of theory and numerical simulations of density (a) and velocity (b) modulation by ion moving with $\sigma_{v_{z}}$ velocity with respect to uniform electron cloud.

In the next simulation, we used an ion moving with the velocity of $v_{0, z}=\sigma_{v_{z}}$ with respect to the electron beam in the comoving frame, where $\sigma_{v}$ is the characteristic electron's velocity spread in the longitudinal direction for the $\kappa-2$ thermal velocity distribution (1). The comparison with theory is shown in Figs. 4(a) (density modulation) and 4(b) (velocity modulation). Again, our simulations demonstrate good agreement with the theory.

\section{B. Modulation in linear focusing field}

To study the modulation processes for a more realistic electron beam, we applied an artificial linear focusing field. We also significantly increased the computational domain and resolved the entire electron bunch in the transverse direction. Assuming the electrons have Gaussian transverse spatial distribution with the rms beam size given in Table II, we applied open boundary conditions in the transverse directions of the system. Fully resolving the transverse size of the electron beam is important as it is comparable with the transverse Debye length. The length of the electron beam given in Table II is orders of magnitude lager compared to the longitudinal Debye length. Since a single ion is used in simulations, we simulate a longitudinal slice of the electron beam with the size of several Debye lengths, using a uniform electron distribution in the longitudinal direction and the periodic boundary conditions.

We have applied a focusing force to prevent the beam expansion due to the space charge force and thermal velocities of electrons. With focusing, the electron beam distribution does not change and the dependence of the modulation process for the Gaussian distribution of electrons can be investigated. In this study, we have used an electron beam with axially symmetric Gaussian distribution in the transverse plane.
There are two components in the focusing electric field. The first component of the focusing field prevents the thermal expansion of the beam in the transverse direction [15]

$$
\vec{E}_{1}(\vec{r})=\frac{m_{e}}{e} \frac{\sigma_{v}^{2}}{\sigma_{r}^{2}}\left(\vec{r}-\vec{r}_{0}\right),
$$

where $\vec{r}=(x, y)$ is the radial coordinate in transverse plane, $\vec{r}_{0}=\left(x_{0}, y_{0}\right)$ is the center of the Gaussian distribution, $\sigma_{r}$ is the RMS of the Gaussian distribution in both horizontal and vertical directions, and $\sigma_{v}$ is the RMS velocity of the electron distribution. The other component of the focusing electric field compensates the expansion due to the space charge forces. We calculate the electric field created by the Gaussian electron beam and apply an external electric field with the same magnitude and opposite sign. Due to the uniform beam distribution on the longitudinal direction, only the transverse space charge electric field needs to be compensated. For an electron beam with the transverse spatial distribution,

$$
n(x, y)=\frac{1}{2 \pi \sigma_{r}^{2}} e^{-\frac{\left(x-x_{0}\right)^{2}+\left(y-y_{0}\right)^{2}}{2 \sigma_{r}^{2}}}
$$

we obtain the following expression for the space charge field

$$
\vec{E}_{2}(\vec{r})=\frac{q}{2 \pi \varepsilon_{0}\left|\vec{r}-\vec{r}_{0}\right|}\left(1-e^{-\left|\vec{r}-\vec{r}_{0}\right|^{2} / 2 \sigma_{r}^{2}}\right)
$$

where $\vec{r}=(x, y)$ is the radial coordinate in transverse plane, $\vec{r}_{0}=\left(x_{0}, y_{0}\right)$ is the center of the Gaussian distribution, $\sigma_{r}$ is the RMS of the Gaussian distribution in both 


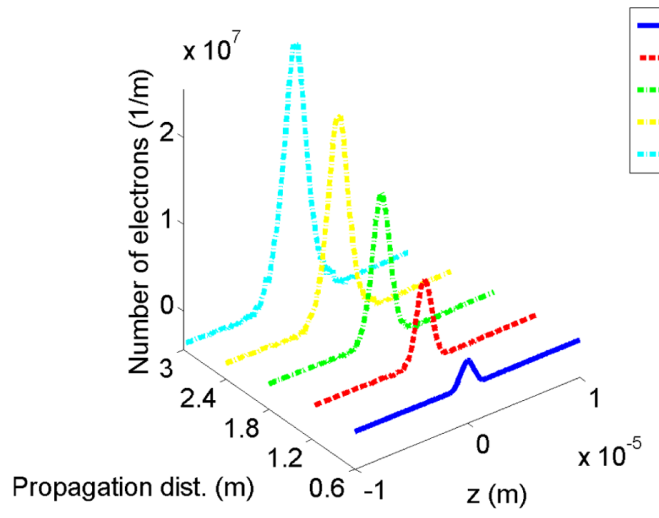

(a) Longitudinal density

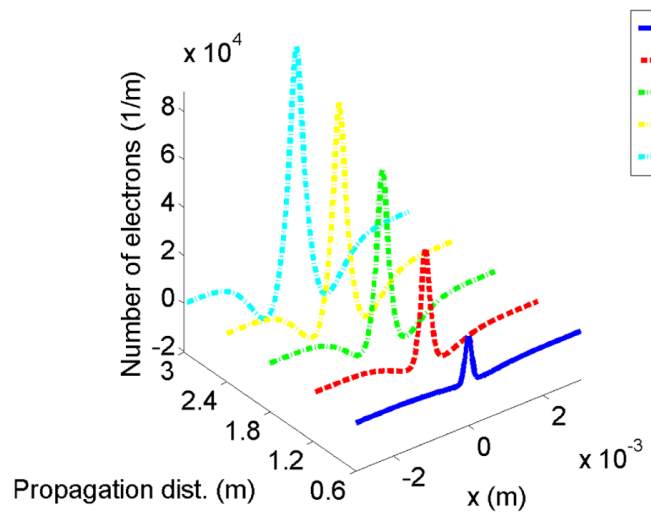

(c) Transverse density

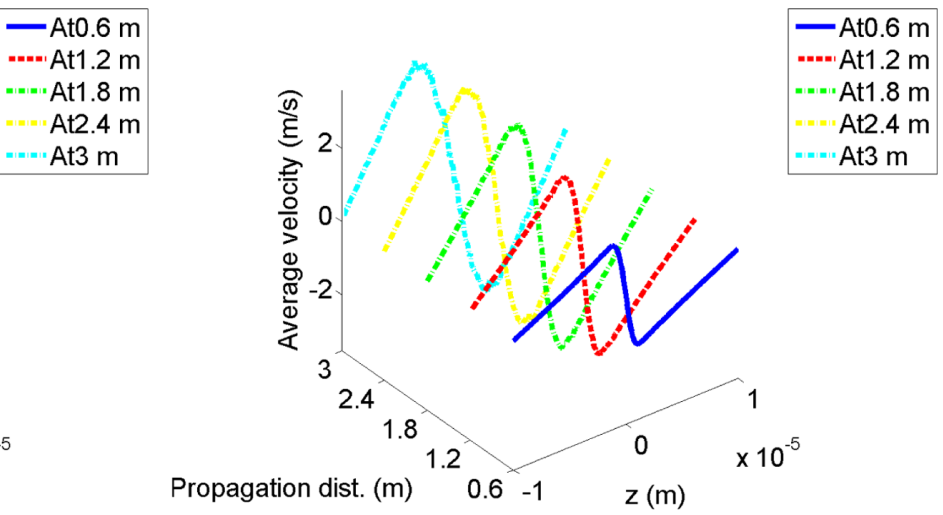

(b) Longitudinal velocity
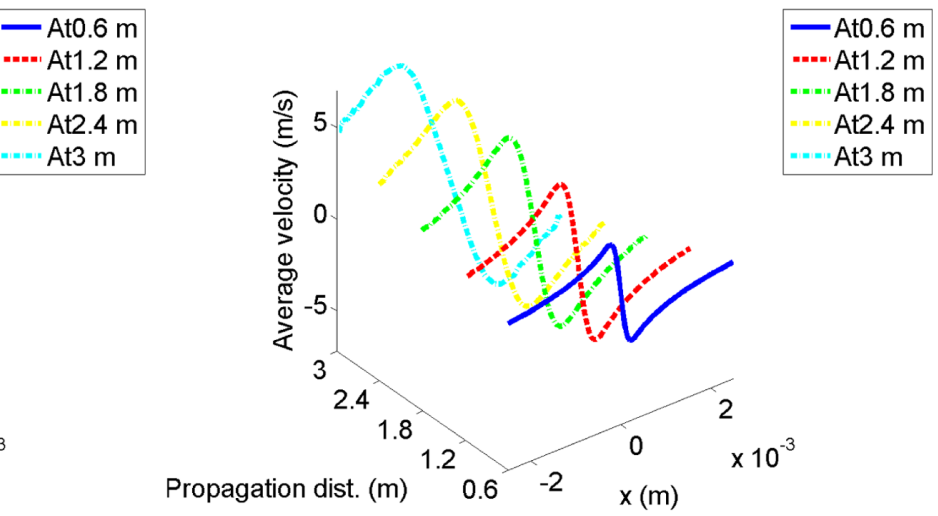

(d) Transverse velocity

FIG. 5. Longitudinal and transverse density and velocity modulation by reference energy ion in the center of the Gaussian electron beam in linear focusing field after $0.6 \mathrm{~m}$ (blue, solid line), $1.2 \mathrm{~m}$ (red, dash line), $1.8 \mathrm{~m}$ (green, dash-dot line), $2.4 \mathrm{~m}$ (yellow, dash-dot line), and $3 \mathrm{~m}$ (cyan, dash-dot line) of copropagation with electrons.

horizontal and vertical directions, and $q$ is electron beam's line charge density. The final focusing field, used in simulations presented in this section, is obtained by combining Eqs. (4) and (6), i.e., $\vec{E}_{\text {total }}=\vec{E}_{1}+\vec{E}_{2}$.

We study first the influence of a stationary ion located in the center of the Gaussian electron beam. The length of modulator is $3 \mathrm{~m}$, which is the copropagation distance of the ion and the electron beam in the laboratory frame. The resulting density and velocity modulations are shown in Fig. 5. Both the longitudinal density and velocity modulation gradually increase with the distance.

All density modulation plots in this paper are presented in the laboratory frame. The velocity modulation values (in $\mathrm{m} / \mathrm{s}$ ) are in the beam frame, and the longitudinal coordinates (in $\mathrm{m}$ ) are scaled to the laboratory frame to be consistent with density plots.

The coordinates on the transverse plane are $x$ in the horizontal direction and $y$ in the vertical direction. Since the distribution of the electron beam holds the radial symmetry in the transverse plane, we typically choose $x$ direction to visualize the transverse modulation. The transverse density modulation [shown in Fig. 5(c)] and the transverse velocity modulation [shown in Fig. 5(d)] are similar to the corresponding quantities in the longitudinal direction. However, regions with negative density modulation, i.e., valleys, appear at the two sides of the main modulation peak, which are absent in the longitudinal modulation profile. We use meter as the domain size unit on the plots instead of the Debye length, because the Debye length depends on the electron density and varies across the Gaussian electron beam.

Applying the principle of superposition, we can compute the modulation process due to the whole ion beam using results of single ion simulations. To obtain the necessary data, we repeat the single ion simulations using various locations and velocities for the ion. Specifically, we place the ion at the distances of $0.5 \sigma_{x}, 1.0 \sigma_{x}, 1.5 \sigma_{x}, 2.0 \sigma_{x}$ off the center of the Gaussian electron beam along $x$ direction, where $\sigma_{x}$ is the RMS beam size. In a series of separate tests, we have rigorously verifies the applicability of the superposition principle for $\mathrm{CeC}$ simulations. Figure 6(a) shows the density and velocity modulation (in the longitudinal and 


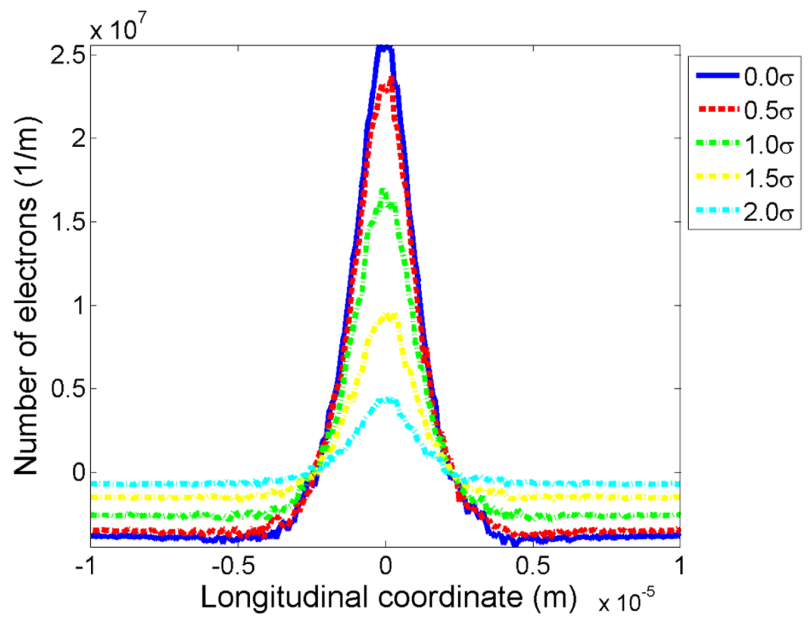

(a) Longitudinal density

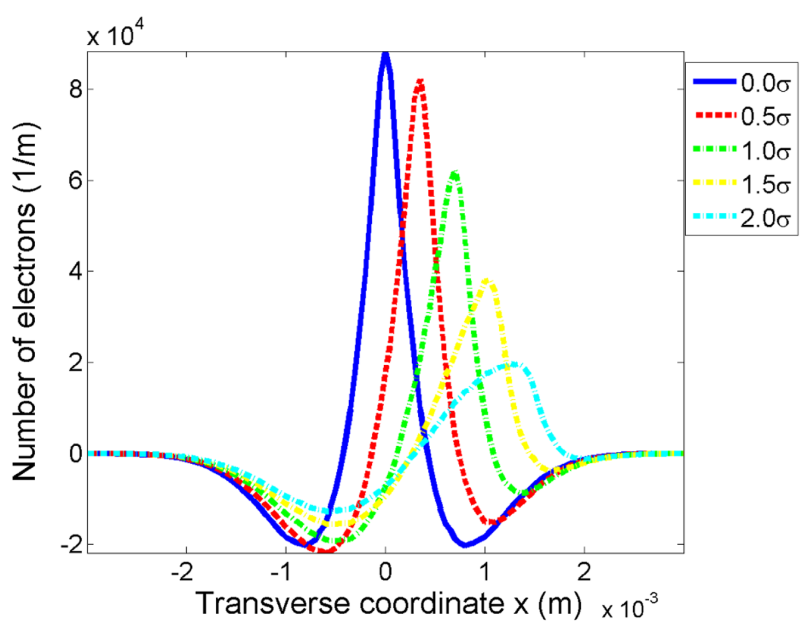

(c) Transverse density

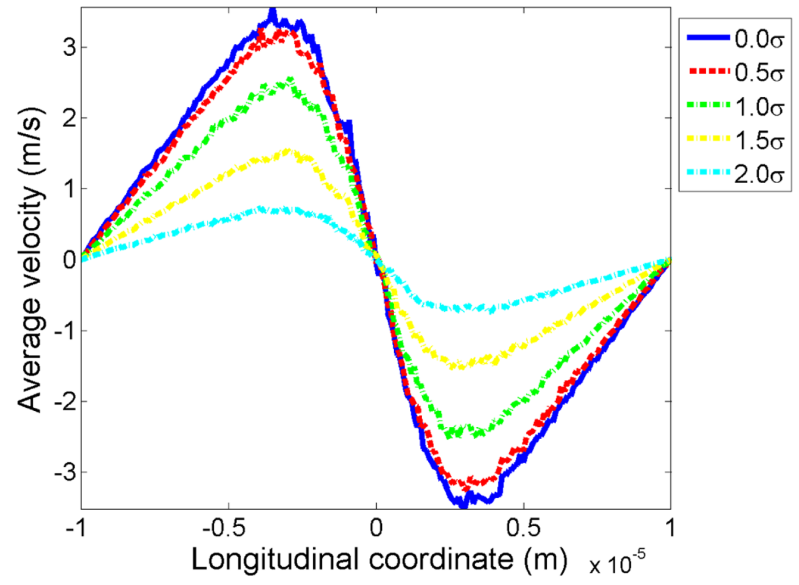

(b) Longitudinal velocity

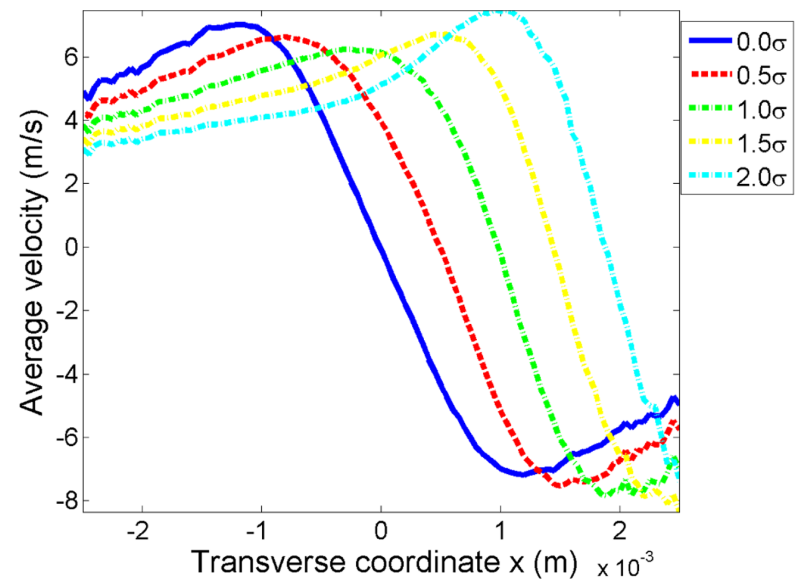

(d) Transverse velocity

FIG. 6. Density and velocity modulation by reference energy ion with various distances off the center of the Gaussian electron beam in linear focusing field after $3 \mathrm{~m}$ of copropagation with electrons.

transverse directions) for various transverse positions of the ion. The longitudinal density modulation in Fig. 6(a) shows a reduction of the modulation amplitude when the ion moves towards the edge of the beam. This can be explained by the reduction of the electrons density. Similar reductions are also visible in the longitudinal velocity modulation in Fig. 6(b). We also observe a loss of symmetry in the transverse distributions of the modulated electron density for off-center trajectory of the ion [see Figs. 6(c) and 6(d)].

2D plots of the density modulation for three transverse positions of the ion are shown in Fig. 7(a). When the ion is at the center of the Gaussian electron beam [Fig. 7(a)], the density modulation is symmetric. For the ion $1 \sigma_{x}$ away from the center, the density of the background electrons drops to approximately $60.6 \%$ of the peak density at the center, and the magnitude of density modulation is reduced to $65.5 \%$. When the ion is at $2 \sigma_{x}$ off the center, where the background electron density is only $13.5 \%$ of the peak density, the density modulation reduces to $17.8 \%$ of the value obtained for the central location of the ion, and the loss of symmetry becomes more obvious. Hence, we can conclude that the change of the modulation amplitude is roughly proportional to the change of the background electron density. The reduction is most likely due to the dependence of the plasma frequency on the local electron density.

The density modulation rapidly drops toward the edge of the beam, where there are few electrons and less screening of the ion attraction force. The density modulation holds a long tail towards the center of the electron beam, in the direction of increasing electron density.

Figure 8 shows a 2D plot of the density modulation in the longitudinal and transverse directions by a stationary ion at the center of the Gaussian electron beam. The computational domain sizes are different in $x$ and $z$ directions, because the Debye lengths in the transverse and longitudinal have different length scales, as is shown in Table II. 


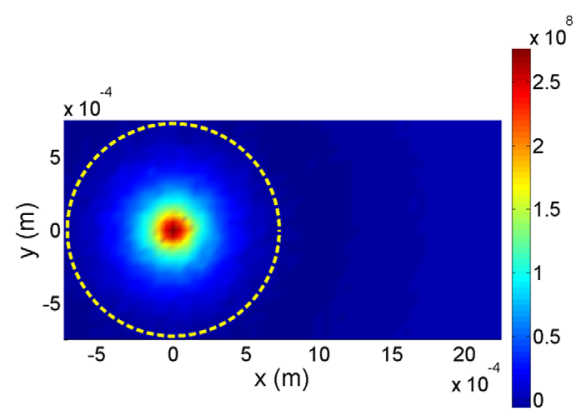

(a) Ion at center

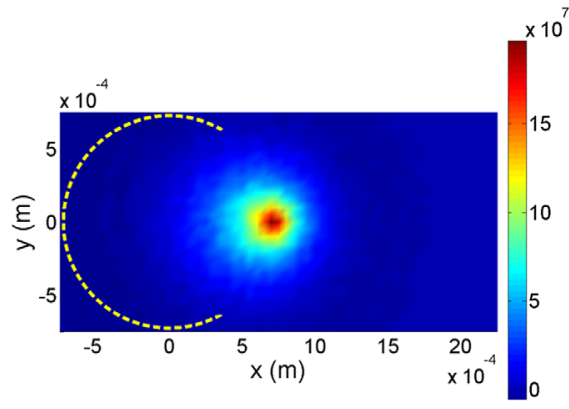

(b) Ion at $x=1 \sigma$

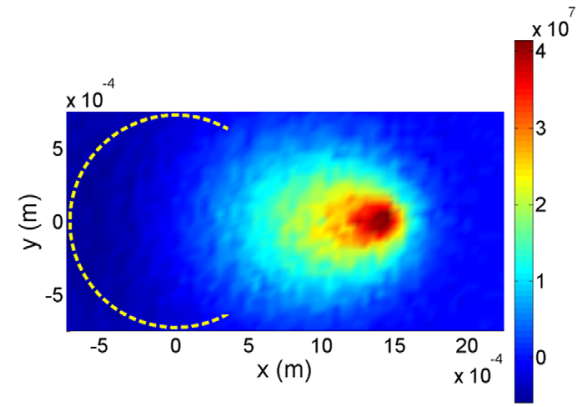

(c) Ion at $x=2 \sigma$

FIG. 7. 2D ( $\mathrm{x}$ and $\mathrm{y}$ ) plots of transverse density modulation of electrons at the exit of the modulator, measured in numbers of electrons per square meters, by resting ion at three transverse positions inside the Gaussian electron beam. The dashed yellow circle indicates the RMS size of Gaussian distribution of the electron beam, which is centered at the center of the beam.

Besides the locations of the ion, its velocity also affects the modulation process. We performed the modulator simulations using an ion moving with velocity $v_{0, z}=1 \cdot \sigma_{v_{z}}$ with respect to the electron beam in the comoving frame, see Eq. (1). We compare the results of the modulation process using a stationary ion and a moving ion, both initially located at the center of the Gaussian electron beam in Fig. 9. The nonsymmetry is observed in Fig. 9(c), with a sharp gradient in the direction of the ion velocity and a long tail in the opposite direction. The longitudinal velocity modulations using a stationary ion [Fig. 9(b)] and a moving ion [Fig. 9(d)] show consistent results. The total number of electrons in the longitudinal density modulation due to the moving ion [shown in Fig. 9(c)] is the same compared to the stationary ion [in Fig. 9(a)]. This is expected as an ion moving in the longitudinal direction experiences a constant background electron density.

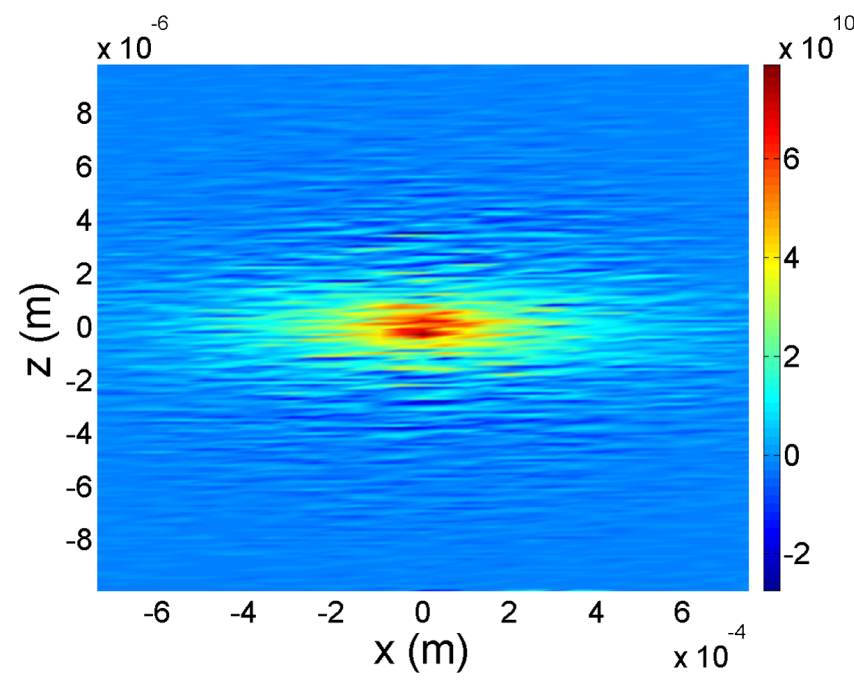

FIG. 8. 2D (x and $\mathrm{z}$ ) plot of density modulation, measured in numbers of electrons per square meters, by stationary $\left(v_{0, z}=0\right)$ ion in the center of the Gaussian electron beam.

\section{Modulation in quadrupole beam line}

In reality, the $\mathrm{CeC}$ experiment at $\mathrm{BNL}$ is using a beam line with four quadrupoles, shown in Fig. 10, as the modulator section. Parameters of the modulator section lattice are listed in Table III. We used a Gaussian axisymmetric distribution of electron beam at the entrance of the modulator section with $\beta_{x, y}=4.2 m, \alpha_{x, y}=0$ and normalized emittance $5 \pi \mathrm{mm}$ mrad.

Magnetic field in the quadrupole is described as

$$
\begin{aligned}
B_{y} & =G(s) \cdot x \\
B_{x} & =G(s) \cdot y
\end{aligned}
$$

where $G$ for hard-edge quadrupoles, which we used in our simulations, is a step function with constant value inside the quadrupole and zero outside it. This magnetic field has infinite derivative at the edges of the quadrupole but provide very accurate model for simulations. As an alternative, we used analytical expressions for the quadrupole magnet field with fringes (as derived in [17]) for our simulations and found the difference from using the hardedge quadrupoles is negligibly small.

We found that space-charge effects are important for the beam dynamics, affecting the beam envelope. In the code SPACE, space charge effects can be turned on and off. We benchmarked our simulation using a well-known beamoptics code MAD-X [18], which does not take into account the space charge. In addition, we have also compared these results with simulations using the code IMPACT-T [19] with the space charge module that also can be turned off. The comparison of $\beta$-function evolutions in the modulator section obtained by MAD-X and SPACE and IMPACT-T codes without space charge is summarized in Fig. 11(a). The corresponding results with the space charge in SPACE and IMPACT-T are presented in Fig. 11(b). The ratio between vertical and horizontal beam sizes in the $\mathrm{CeC}$ modulator is plotted in Fig. 11(c).

One can see that space charge significantly affects the vertical $\beta$-function at the exit of the modulator section. 


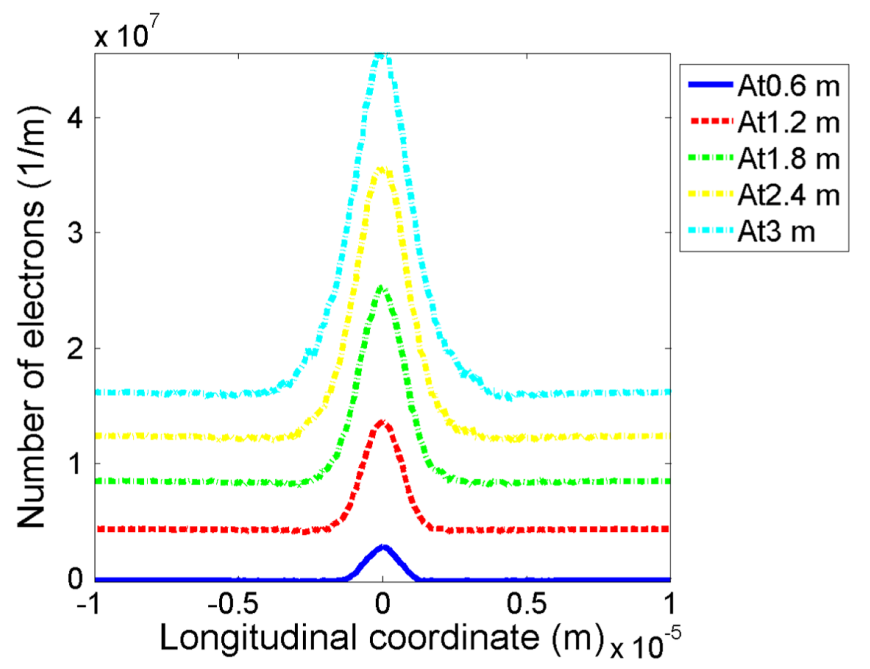

(a) Longitudinal density, stationary ion

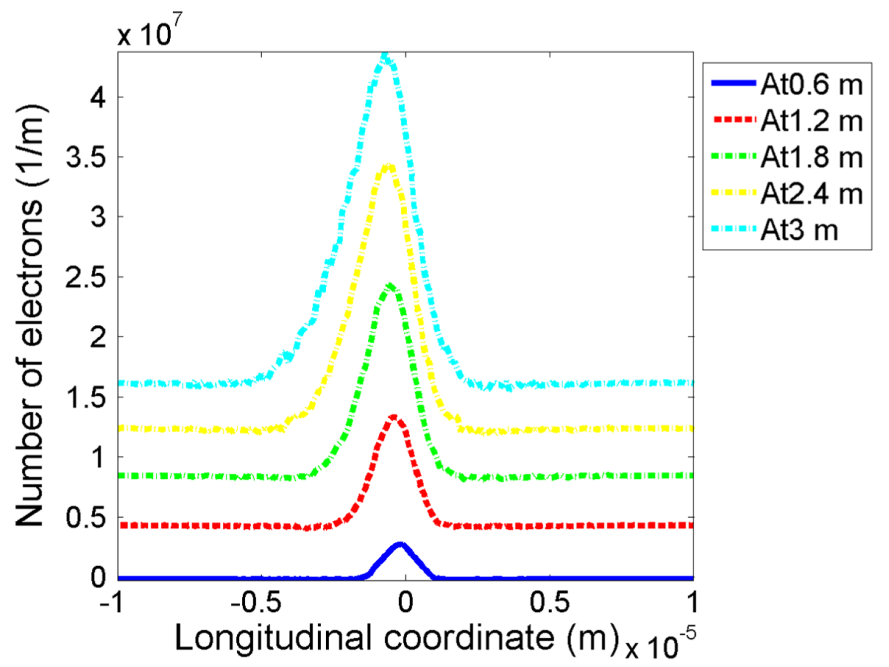

(c) Longitudinal density, moving ion

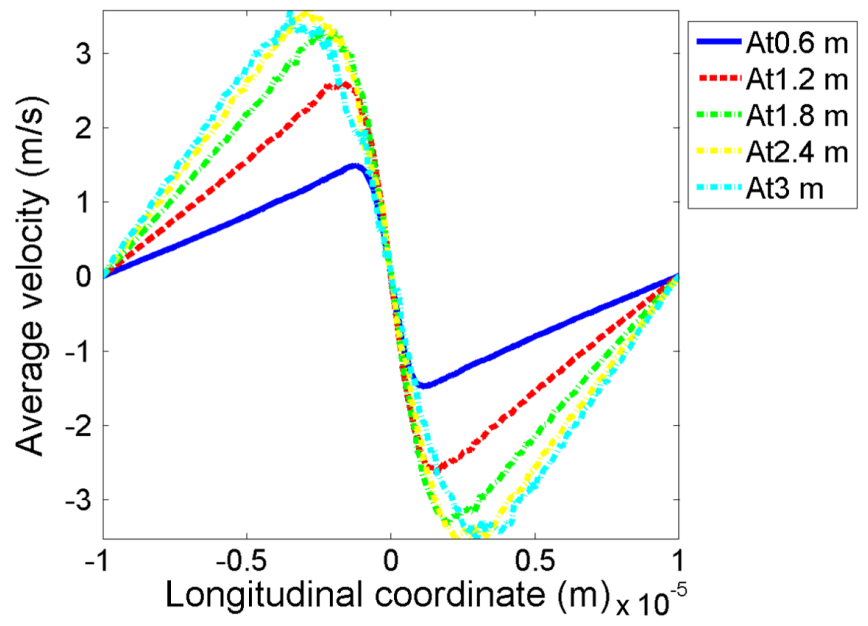

(b) Longitudinal velocity, stationary ion

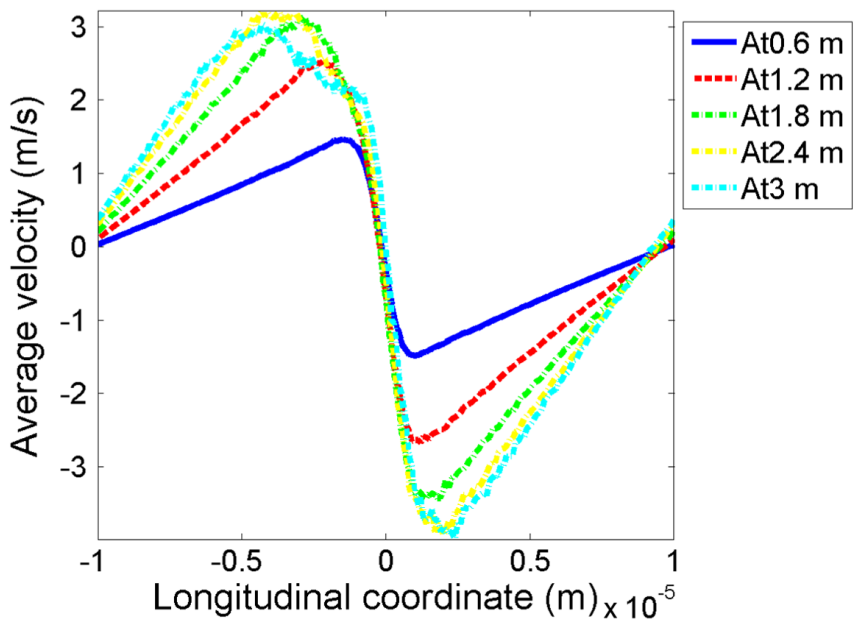

(d) Longitudinal velocity, moving ion

FIG. 9. Longitudinal density and velocity modulation by stationary and moving ions, transversely located in the center of the Gaussian electron beam after $0.6 \mathrm{~m}$ (blue, solid line), $1.2 \mathrm{~m}$ (red, dash line), $1.8 \mathrm{~m}$ (green, dash-dot line), $2.4 \mathrm{~m}$ (yellow, dash-dot line), and $3 \mathrm{~m}$ (cyan, dash-dot line) length of copropagation.

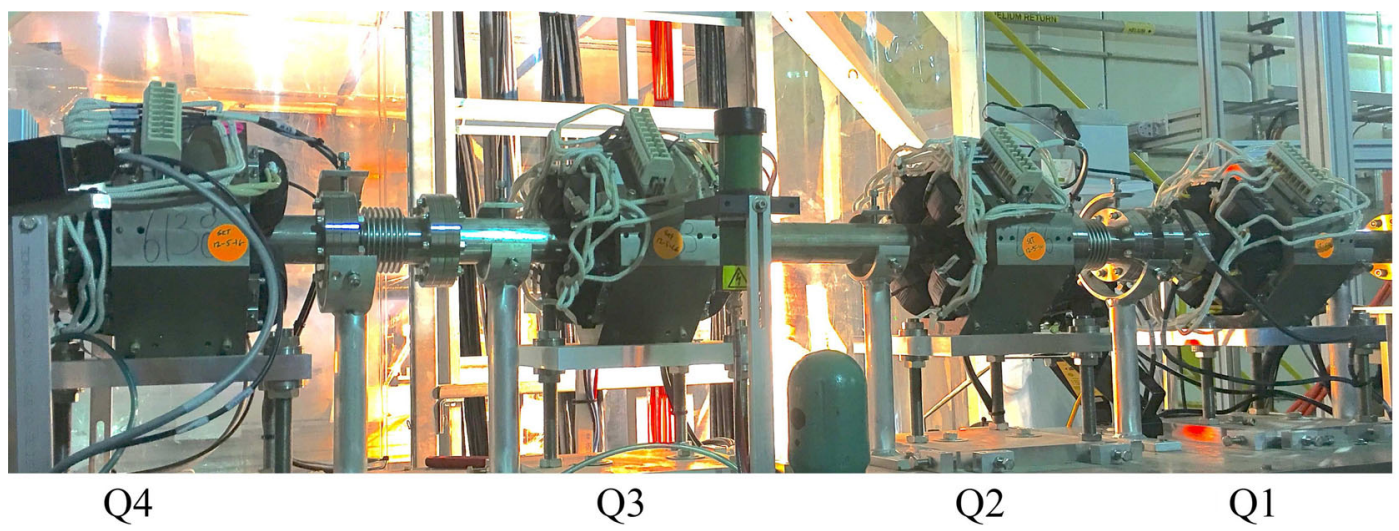

FIG. 10. CeC modulator section comprising four quadrupoles. The electron beam propagates from right to left. 
TABLE III. Lattice of the modulator section.

\begin{tabular}{lcc}
\hline \hline Element & Length, $\mathrm{m}$ & Magnetic field gradient, T/m \\
\hline Drift & 0.4245 & \\
Q1 quadrupole & 0.157 & 0.5528 \\
Drift & 0.393 & -0.6220 \\
Q2 quadrupole & 0.157 & \\
Drift & 0.393 & -0.0511 \\
Q3 quadrupole & 0.157 & \\
Drift & 0.393 & 0.6072 \\
Q4 quadrupole & 0.157 & \\
Drift & 0.7685 & \\
\hline \hline
\end{tabular}

Small differences between the SPACE and IMPACT-T simulations at the quadrupole exit can not be attributed solely to the space charge. In the case of the space charge turned off, SPACE agrees well with MAD-X, but IMPACT-T gives smaller values of $\beta_{y}$ compared to both codes. In addition, IMPACT-T did not obtain perfectly matched $\beta_{x}$ and $\beta_{y}$ at the end of the modulator. Withe the space charge turned on, the differences are similar but slightly amplified. Open boundary conditions were used by both codes.

Space charge was included in the simulation of the modulation induced by the ion. The space charge forces act only on the electron beam. As their effect on an offcentered ion is negligibly small, we did not include the effect of the electron beam space charge on the ion motion. For the same reason, the effect of the quadrupole field on the motion of the ion was also neglected. Results of our simulations are summarized in Figs. 12 and 13. While the evolution of the longitudinal density and the velocity modulation are qualitatively very similar to those observed for a uniform linear constant focusing, the density and velocity modulation in the transverse direction has new

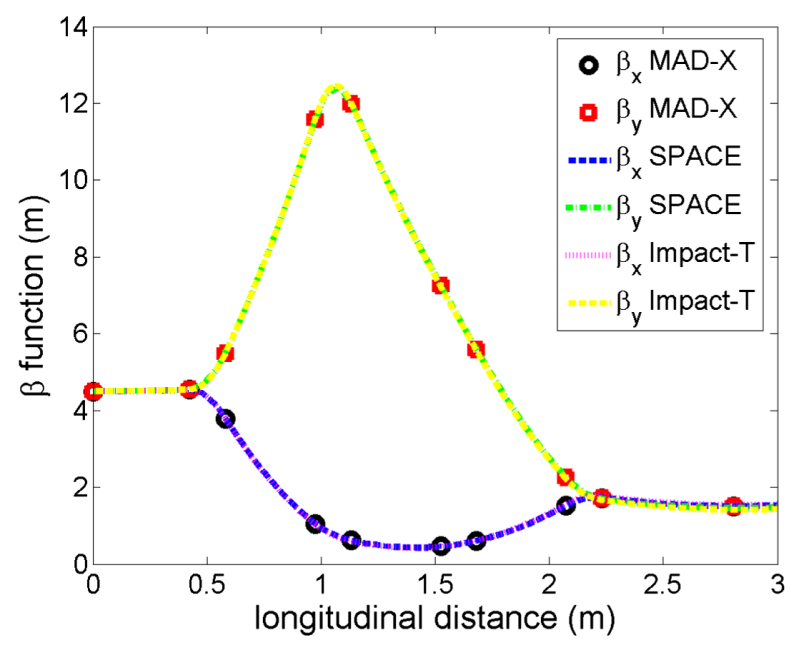

(a)

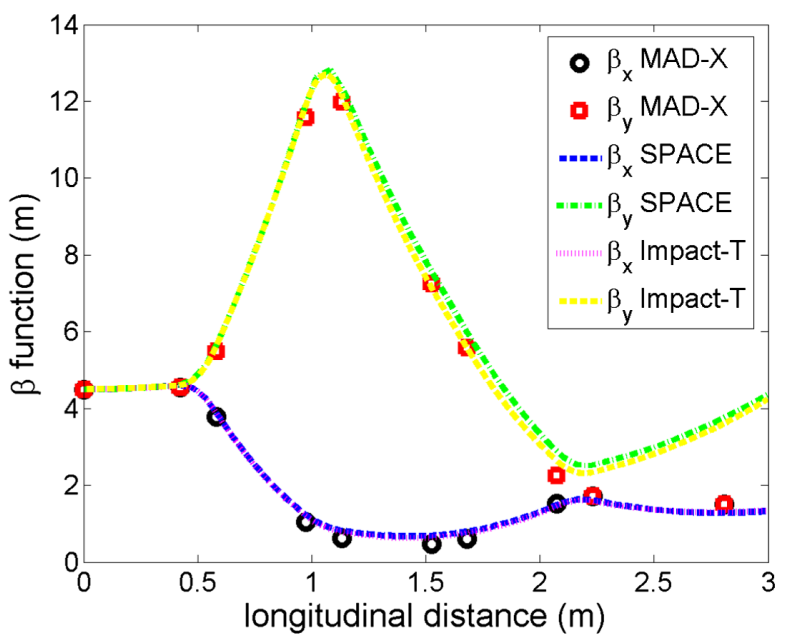

(b)

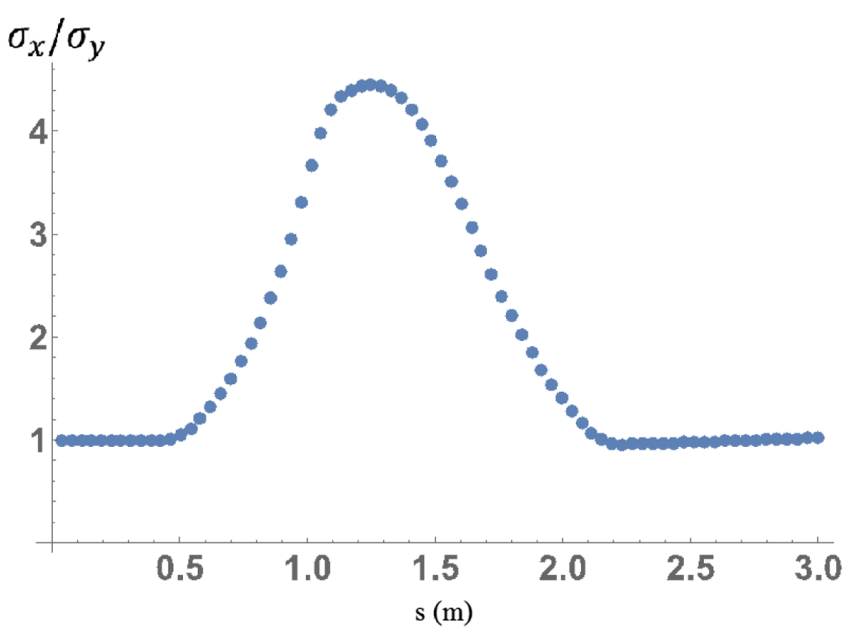

(c)

FIG. 11. (a) Evolution of $\beta$-functions in the modulator section obtained by MAD-X code (red dots and black dots), SPACE with the space charge turned off (blue and green dash-lines), and IMPACT-T with the space charge turned off (magenta and yellow dash-lines). (b) Same as in (a), but the space charge was turned on in both SPACE and IMPACT-T codes. (c) Ratio between vertical and horizontal beam sizes in the $\mathrm{CeC}$ modulator. 


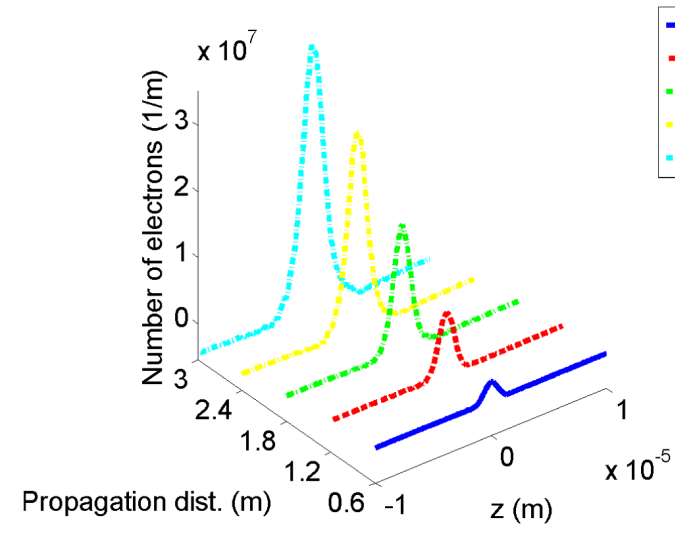

(a) Longitudinal density, 3D

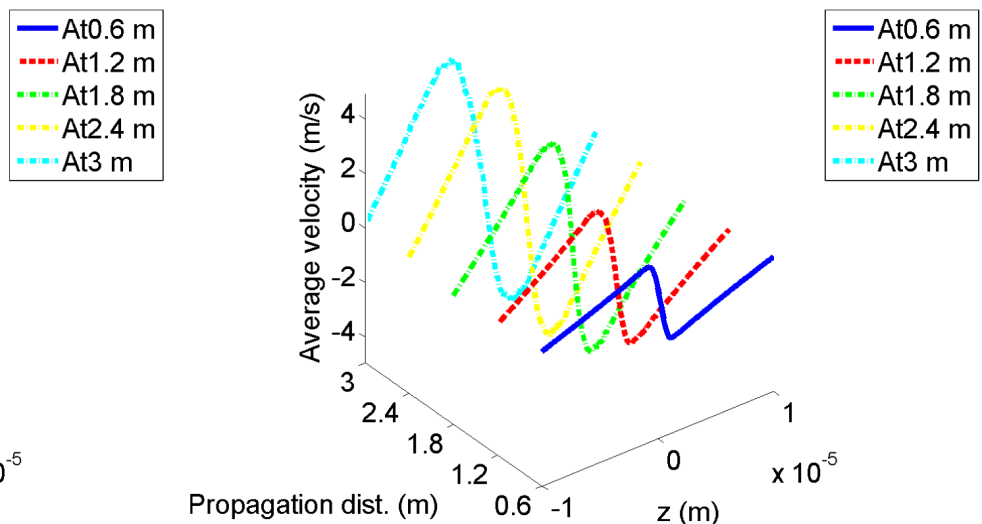

(b) Longitudinal velocity, 3D

FIG. 12. Longitudinal density and velocity modulation by stationary ion on axis of the Gaussian electron beam in the CeC modulator: blue, solid line — after $0.6 \mathrm{~m}$, red, dash line — after $1.2 \mathrm{~m}$, green, dash-dot line_-after $1.8 \mathrm{~m}$, yellow, dash-dot line—after $2.4 \mathrm{~m}$, and cyan, dash-dot line-after $3 \mathrm{~m}$ of copropagation through the modulator.

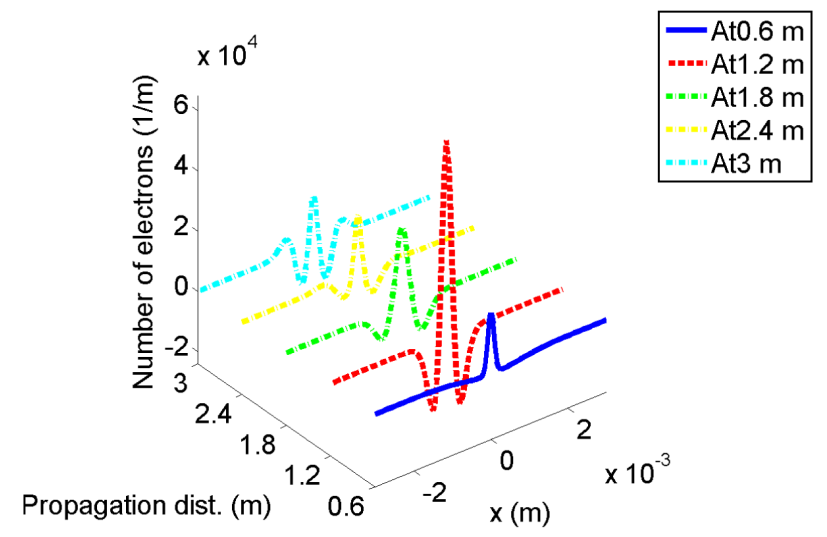

(a) Transverse (x) density, 3D

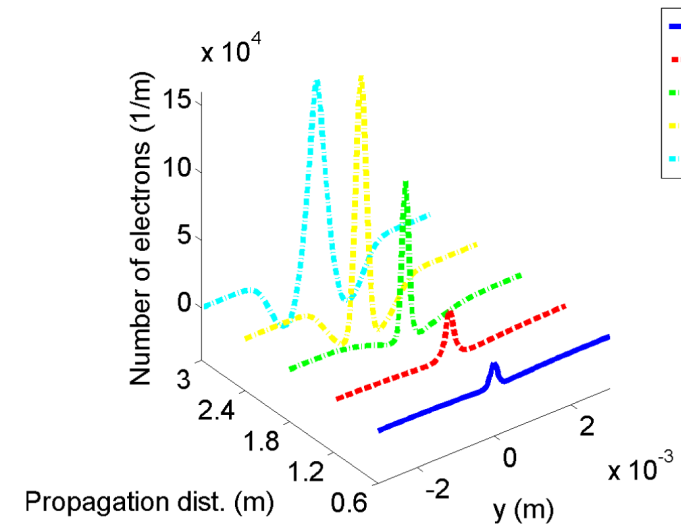

(c) Transverse (y) density, 3D

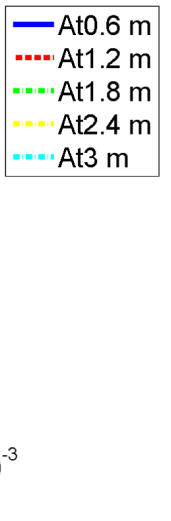

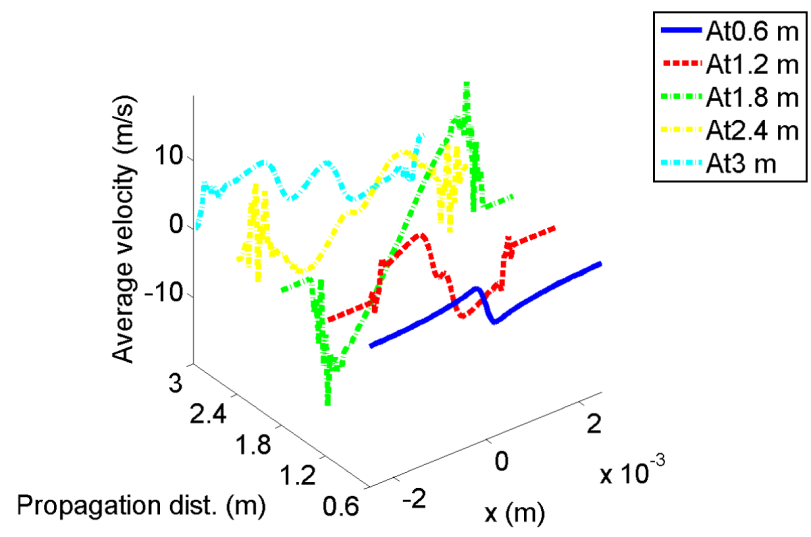

(b) Transverse (x) velocity, 3D

Propagation dist. (m)

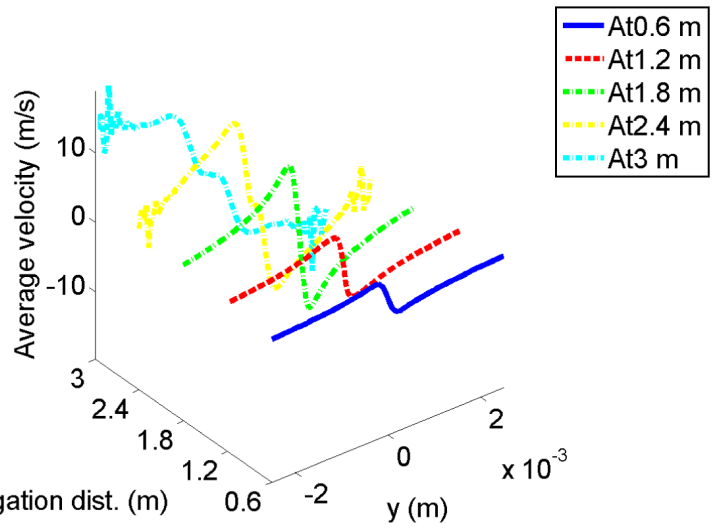

(d) Transverse (y) velocity, 3D

FIG. 13. Transverse density and velocity modulation by stationary ion on axis of the Gaussian electron beam in the CeC modulator: blue, solid line — after $0.6 \mathrm{~m}$, red, dash line — after $1.2 \mathrm{~m}$, green, dash-dot line—after $1.8 \mathrm{~m}$, yellow, dash-dot line — after $2.4 \mathrm{~m}$, and cyan, dash-dot line-after $3 \mathrm{~m}$ of copropagation through the modulator. 
features, which as we show below originated from strong quadrupole focusing and variation of the electron beam sizes along the beam line.

Specifically, in the longitudinal direction, we notice a slight asymmetry towards the forward direction. This asymmetry is explained by the energy conservation in the magnetic field of quadrupoles: e.g., the average longitudinal momentum of the electrons executing betatron oscillation in the quadrupole channel is slightly lower than those on axis and whose velocity is equal to that of the ion. In other words, an ion traveling on-axis is slightly faster than the average electron beam having the same relativistic $\gamma$-factor.

In contrast, the density and velocity modulation in the transverse direction (especially in horizontal, $\mathrm{x}$, direction) varies rather dramatically from a gradual smooth evolution observed for the uniform linear constant focusing [compare Figs. 5(c) and 5(d) with Figs. 13(a) and 13(b)]. As can be seen from Fig. 13, the initial interaction with the ion (at $s=0.6 \mathrm{~m}$ ) introduces negative correlation between the position and velocity of electrons:

$$
\begin{aligned}
& \left\langle x \delta x^{\prime}\right\rangle<0 \\
& \left\langle y \delta y^{\prime}\right\rangle<0
\end{aligned}
$$

where $\delta x^{\prime}=\frac{m v_{x}}{p c}, \delta y^{\prime}=\frac{m v_{y}}{p c}$ are average angular kicks (in the laboratory frame) received by an electron through attracting the ion located at $x=y=0$. Here $m$ is the mass of electron, $c$ is the speed of the light and $p$ is the relativistic momentum of the electron. Then the behavior of the modulation in the $x$-direction exhibits the following phenomena: the density modulation reaches its peak at about $s=1.2 \mathrm{~m}$ and then the central values start falling while the side wings are clearly developing by the beam reaching $2.4 \mathrm{~m}$ and $3 \mathrm{~m}$. Even more remarkable is that at $1.8 \mathrm{~m}$, we can see a clear change of the sign in the velocity modulation.

While only the longitudinal modulation in the modulator plays important role in the coherent cooling process, understanding the nature of the transverse density and velocity evolution is important to confirm that our code correctly computes the physics of all process. This is the reason for our detailed studies of this phenomenon. At $s=1.2 \mathrm{~m}$, the horizontal density modulation reaches its maximum, which could be explained by the fact that the horizontal size $\sigma_{x}=\sqrt{\beta_{x} \varepsilon_{n} / \gamma}$ reaches minimum around that point. What is unusual is a flattening of the velocity modulation near $x=0$ and a ripple at $x \sim \pm 1.5 \mathrm{~mm}$. Still, the most dramatic is the reversal of the sign of the velocity modulation at $s=1.8 \mathrm{~m}$ and the consequent reduction of the central peak in the density. It means that the correlation $\left\langle x \delta x^{\prime}\right\rangle$ reversed the sign and the modulation cloud was not expanding in size. It typically happens for plasma oscillations at $3 / 4$ of the plasma oscillation in a normal (constant density plasma) conditions. At the same time, the vertical density gradually increases till $s=2.4 \mathrm{~m}$ and only after that slightly falls. Similarly, the velocity modulation evolves regularly and only at $s=3$ m one can notice some flattening at $y=0$. It is worth noting that similar flattening is clearly seen in $x$-direction as $s=1.2 \mathrm{~m}$.

It is remarkable that the evolution of the modulation in all three directions has significant differences: (a) in the longitudinal $z$-direction, the evolution is nearly identical to that in the infinite plasma, and reaches its peak at about $1 / 2$ of the plasma oscillation period ${ }^{1}$; (b) in the vertical direction, with the vertical size being significantly larger than the horizontal size in the central part of the modulator, the peak of the density is reached at about $80 \%$ along the distance of the modulator, and (c) in the horizontal direction, this happens at $40 \%$ of the modulator length.

The explanation of this phenomenon is two-fold, originating from strong asymmetric quadrupole focusing in the $\mathrm{CeC}$ modulator. First, as we mentioned before, the transverse beam sizes of electron beam are comparable with the Debye radii. Since electrons closer to the ion are moving faster than those at the periphery, they are reaching the saturation density around the ion earlier than at $1 / 4$ of the nominal plasma oscillation, and the velocity reversal happens earlier than at $1 / 2$ of the "oscillation." This is the most reasonable explanation of the flattening of the velocity modulation near $x=0$ and $y=0$. It is also the most likely reason of the expanding wave responsible for the oscillating wing in the density modulation. This phenomenon has similarity with the presence of waves in shielding of a moving charged particle in a confined electron plasma [8].

Explanation for a significant 2.5 -fold difference in the reaction time for horizontal and vertical directions is most likely related to a very strong focusing (compressing) of the electron beam in the horizontal direction and its expansion in the vertical direction, as is clearly demonstrated in Fig. 11. At $s=1.2 \mathrm{~m}$, where the horizontal density peaks, the vertical size is 4 times larger than the horizontal. Assuming that the distribution of the density modulation has the same shape as the transverse beam, we can estimate the ratio between the horizontal and vertical repelling electric fields as [20]:

$$
\frac{E_{x}}{E_{y}} \cong \frac{\sigma_{y}}{\sigma_{x}}
$$

i.e., the repulsion in the horizontal direction is significantly stronger for the most of the path in the modulator, and

\footnotetext{
${ }^{1}$ It is important to note that there is no uniform definition of plasma frequency for nonuniform electron beam with Gaussian distribution. Only a rough estimate using the core of the beam density can be done in this case. The beam sizes and the length of the modulator were selected to have approximately $1 / 2$ of the plasma oscillation in the plasma with uniform density of the average density of the beam core.
} 
especially in its middle. This strong horizontal quadrupole focusing is likely the most significant contributor to the early sign reversal in the velocity modulation and $\left\langle x \delta x^{\prime}\right\rangle$ correlation.

The other apparent contributor is the betatron motion of the particles. We designed a simple model to show that the betatron oscillation in a strong focusing beam line can reverse sign of $\left\langle x \delta x^{\prime}\right\rangle$ correlation. First, we observe that $\left|\delta_{x}^{\prime}\right| \ll \sigma_{x^{\prime}}$, i.e., the perturbation of electron's motion by the ion is negligibly small when compared with a typical amplitude of betatron oscillations. Thus, let us consider that there is an initial nonzero (e.g., negative) correlation at $s=0$ between electron position $x_{o}$ and angular kick to $\left.\delta x_{o}^{\prime}=\left\langle\delta x^{\prime}\left(x_{0}\right)\right\rangle\right)$ electrons locates at $x_{o}$ from the on-axis ion:

$$
\left\langle x_{o} \delta x_{o}^{\prime}\right\rangle=-\varepsilon, \varepsilon>0 .
$$

The averaging for the kick is taken over the rest of the coordinates and angles, i.e., $\left\langle x_{o}^{\prime} \delta x_{o}^{\prime}\right\rangle=0$. Let us consider a linear $2 \times 2$ symplectic horizontal transport matrix typical for quadrupole channel:

$$
\left(\begin{array}{l}
x(s) \\
x^{\prime}(s)
\end{array}\right)=\left(\begin{array}{ll}
a(s) & b(s) \\
c(s) & d(s)
\end{array}\right)\left(\begin{array}{c}
x_{o} \\
x_{o}^{\prime}
\end{array}\right), \quad a d-b c=1
$$

which naturally applies to the kick induced at $s=0$ :

$$
\left(\begin{array}{c}
\delta x(s) \\
\delta x^{\prime}(s)
\end{array}\right)=\left(\begin{array}{cc}
a(s) & b(s) \\
c(s) & d(s)
\end{array}\right)\left(\begin{array}{c}
0 \\
\delta x_{o}^{\prime}
\end{array}\right)
$$

Then the correlation $\left\langle x(s) \delta x^{\prime}(s)\right\rangle$ at an azimuth s can be easily calculated to be

$$
\begin{aligned}
x & =a x_{o}+b x_{o}^{\prime} \\
\delta x^{\prime} & =d \delta x_{o}^{\prime} \\
\left\langle x \delta x^{\prime}\right\rangle & =a d \cdot\left\langle x_{o} \delta x_{o}^{\prime}\right\rangle \\
& =-a d \cdot \varepsilon
\end{aligned}
$$

where we neglected second order terms in $\varepsilon$. Equation (13) simply states that the correlation sign between electrons' position and velocity can be reversed if the product of the diagonal matrix elements becomes negative. In contrast with the case of constant focusing or defocusing, when $a(s) \equiv d(s)$, and such a reversal of the sign is impossible, strong focusing allows for this to happen. Actually, our lattice provides for such a reversal (for the modulation originated at the beginning of the modulator) at s interval of $\{1.6 \mathrm{~m}-2.2 \mathrm{~m}\}$, as it is illustrated in Fig. 14. Naturally, the area where the reversal happens depends on the origin of the modulation, but our studies showed that it always occurs in the horizontal direction.

Thus, we can conclude that a combination of asymmetric repelling forces and the reversal of $\left\langle x(s) \delta x(s)^{\prime}\right\rangle$ correlations is responsible for unusual evolution of the transverse

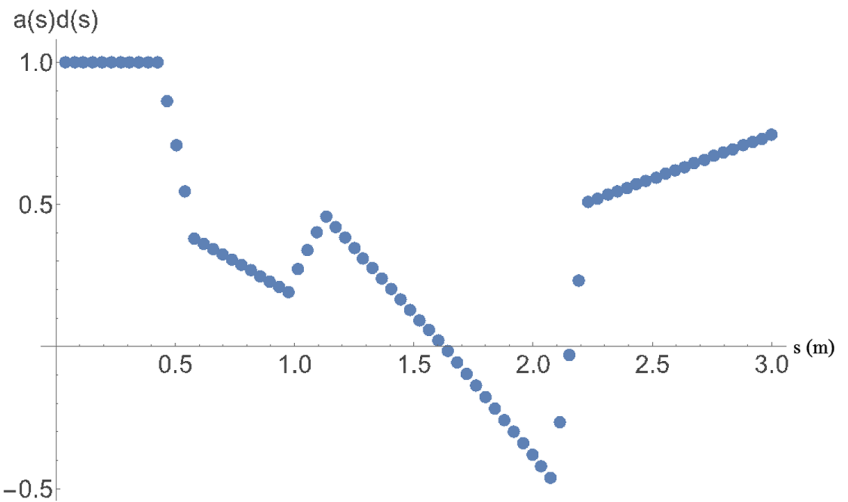

FIG. 14. Product of diagonal elements of horizontal transport matrix as function of length along the length of the $\mathrm{CeC}$ modulator.

density and velocity modulation, both originating from strong quadrupole focusing in the modulator beam line. Detailed studies of the motion of individual particles inside the electron beam [21] confirm these conclusions.

\section{Modulation in quadrupole beam line for an off-axis ion}

Similarly to our previous studies, we found that the quadrupole beam line introduces some peculiarities in the modulation processes for an off-axis ion. First, we would like to note that the ion displacement from the electron beam axis, measured in RMS beam sizes at the entrance of the modulator (e.g., where $\beta_{x, y}=4.2 \mathrm{~m}$ ), remains constant while the electron beam size evolves. Figure 15 illustrates the longitudinal density modulation at the end of the modulator $(s=3 \mathrm{~m})$ for ions displaced by $0 \sigma_{x}, 0.5 \sigma_{x}$, $1 \sigma_{x}, 1.5 \sigma_{x}$, and $2 \sigma_{x}$, respectively. As expected, the amplitude of the longitudinal density modulation drops with the ion moving from the electron beam axis to its edge where the electron density is lower. In addition, there is a slight backward-forward asymmetry in modulation signal, which becomes stronger when the ion moves further offaxis [see Fig. 15(b) with zoomed vertical scale]. The reason is that electrons interacting with an off-axis ion execute betatron oscillation, and the length of their trajectory elongates as square of the betatron amplitude, ${ }^{2}$ while their velocity is the same as the ion moving along the straight line.

The evolution of the transverse density modulation in the $x$ direction also becomes more complicated for the off-axis ions. Placing an ion off-axis not only introduces nonlinearity into the space charge effects but also causes moving of the modulation signal, both due to quadrupoles. Because of the larger vertical beam size along the modulator beam line, these effects are less prominent in the $y$-direction and mostly affect the horizontal density modulation.

${ }^{2}$ To be exact, $s=\int \sqrt{1+x^{\prime 2}+y^{\prime 2}} d z \cong \int\left(1+\left(x^{\prime 2}+y^{\prime 2}\right) / 2\right) d z$. 


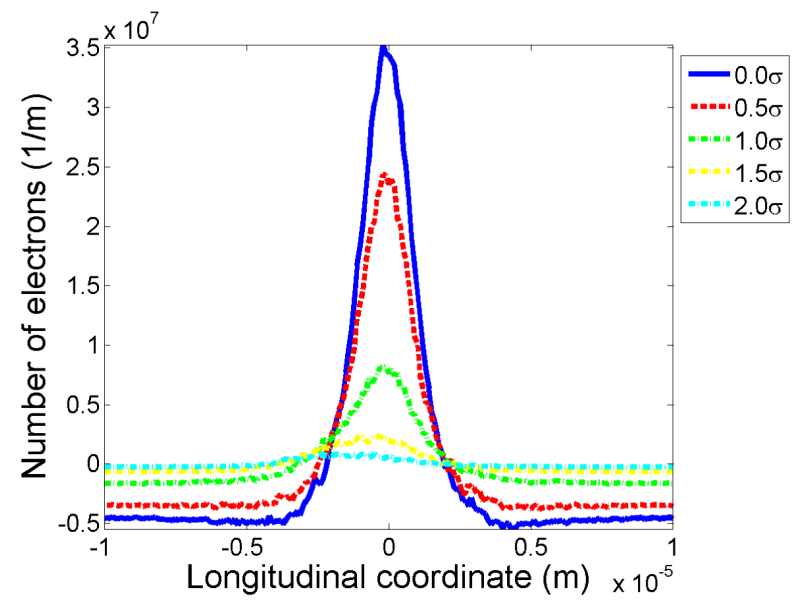

(a) Longitudinal density

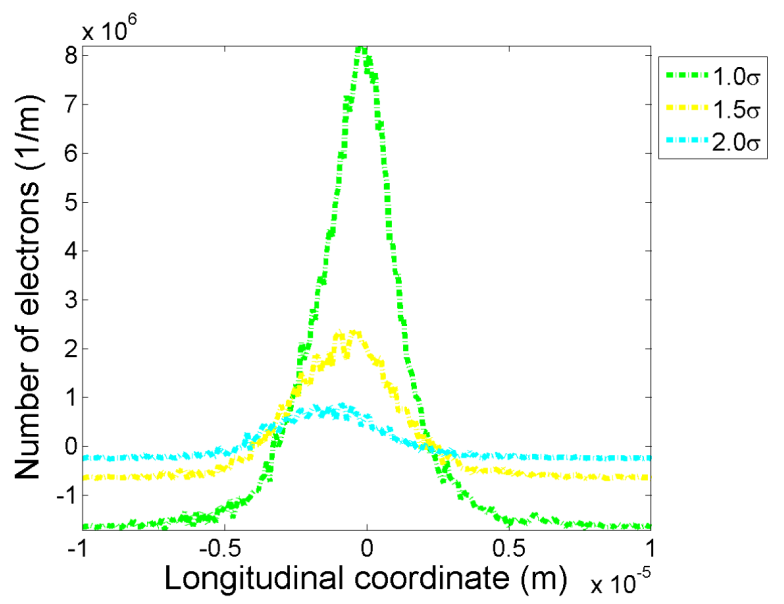

(b) Longitudinal density, zoom in

FIG. 15. Longitudinal density modulation at the end of the modulator by stationary ion with various displacements from the axis in $x$ direction.

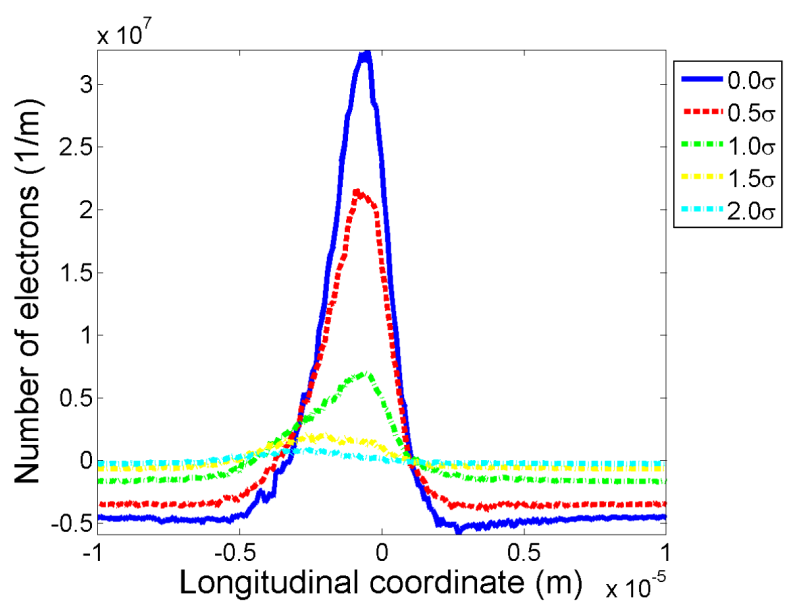

(a) Longitudinal density

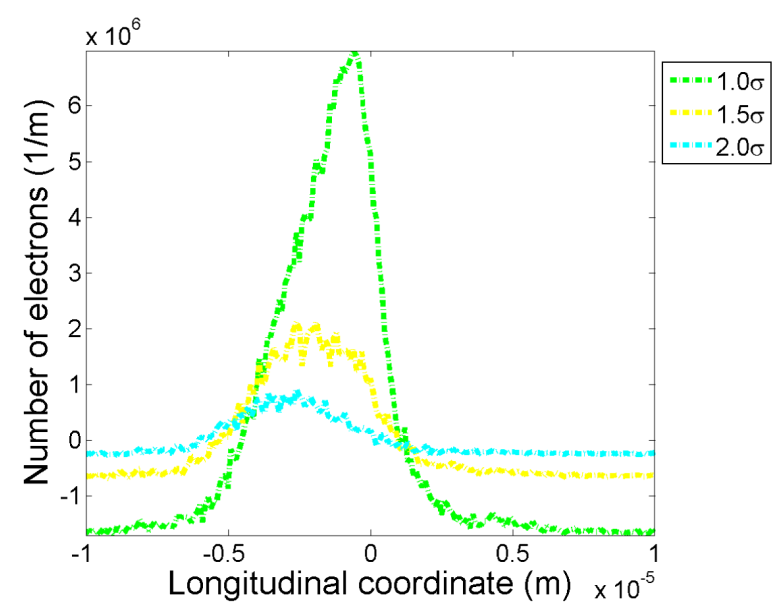

(b) Longitudinal density, zoom in

FIG. 16. Longitudinal density modulation at $s=3 \mathrm{~m}$ in the $\mathrm{CeC}$ modulator induced by the moving ion with $v_{0, z}=\sigma_{v_{z}}$ along the horizontally shifted off-axis trajectory.

As we discussed above, the details of the transverse density modulation, while being of academic interest, are not important for the FEL-based $\mathrm{CeC}$ [1], where the diffraction in the FEL washes away this information. But they can be critical for $\mathrm{CeC}$ based on other amplifiers, for example the microbunching based $\mathrm{CeC}$ [5].

Finally, Fig. 16 summarizes the dependence of the longitudinal density at the exit of the $\mathrm{CeC}$ modulator for an ion moving (in the electron beam comoving frame) with the longitudinal velocity $v_{0, z}=\sigma_{v_{z}}$ along an off-axis trajectory.

\section{E. Impact on the bunching factor}

The most important factor in the amplification (FEL) section of the $\mathrm{CeC}$ experiment is the bunching factor [22]. The bunching factor is defined as

$$
b=\frac{1}{N_{\lambda}} \sum_{k=1}^{N_{\lambda}} e^{i \frac{2 \pi}{\lambda_{\mathrm{opt}}} z_{k}}, \quad \text { for }-\frac{\lambda_{\mathrm{opt}}}{2} \leq z_{k} \leq \frac{\lambda_{\mathrm{opt}}}{2},
$$

where $\lambda_{\text {opt }}$ is the FEL optical wavelength, the summation is over a slice of $\lambda_{\text {opt }}$ wide, centered at the ion location, and $N_{\lambda}$ is the total number of electrons within that slice.

The bunching factor amplitude at the end of a $\mathrm{CeC}$ modulator containing an infinite electron beam and a moving ion can be computed analytically [22]. In Fig. 17, we plotted the dependence of the bunching factor amplitude on the ion velocity. Ion velocity in Fig. 17 is in unit of electron velocity spread, which is fixed at 1e-3 in this study. The same plot contains several numerical simulation points obtained using a finite electron beam in the focusing channel. As theory assumes infinite electron beam with uniform distribution and simulation uses finite 


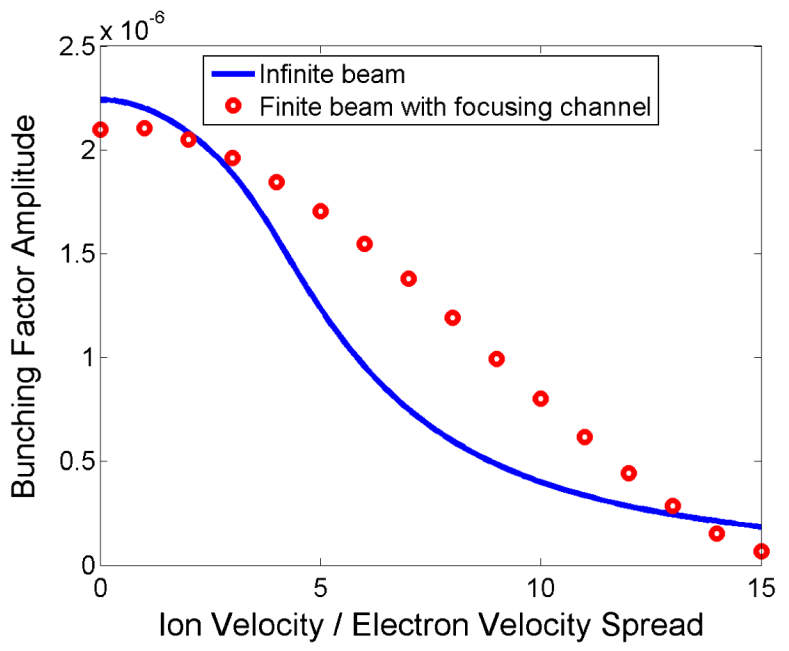

FIG. 17. Dependence of the bunching factor amplitude on the ion velocity at the end of the $\mathrm{CeC}$ modulator, electron velocity spread is fixed at $1 \mathrm{e}-3$ and is used as a normalization constant.

electron beam with normal distribution in a quadrupole beam line, we expect a difference in bunching factor between analytical values and simulation results. Both theory and simulation show the similar trend that bunching factor decreases when the ion velocity increases. This plot demonstrates a strong dependence of the bunching factor amplitude on the ion velocity, and justifies our $\mathrm{CeC}$ modulation studies using moving ions. As the bunching factor also strongly depends on the ion position with respect to the center of the Gaussian electron beam, it was also necessary to perform simulations studies of the modulation with off-centered ions.

\section{CONCLUSIONS AND FUTURE WORK}

Simulating the electron density and velocity modulation by an ion copropagating with a realistic (e.g., finite and nonuniform) electron beam in a realistic modulator (e.g., a quadrupole beam line) represents a very serious challenge. The presence of strong shot noise and the 3D nature of the problem with open boundaries only complicates the problem. We have developed numerical algorithms and performed high fidelity numerical simulations of this process for the modulator section of the coherent electron cooling experiment, which is undergoing commissioning at RHIC collider, BNL. Our tool of choice for the numerical simulations is SPACE, a parallel, relativistic, 3D electromagnetic PIC code. SPACE uses a new highly adaptive particle-in-cloud (AP-Cloud) method in a module for solving the VlasovPoisson equations. We have also developed a self-consistent method for subtracting the short noise using two simulations - with and without presence of the ion - and identical initial distribution of electrons.

We have tested our simulation for analytically solvable systems (uniform plasma with $\kappa-2$ thermal velocity distribution) and have achieved an excellent agreement of simulation results with analytical solutions. We have extended studies to a model case of ion interacting with transversely finite Gaussian beam, artificially stabilized to a stationary shape by necessary external electric fields. It allows us to establish reliable simulations with transversely opened boundary conditions.

Most importantly, we have achieved reliable simulations for a realistic electron beam propagating in the actual modulator system-a 3-meter long quadrupole beam line-built for the $\mathrm{CeC}$ demonstration experiment. We discovered that while the longitudinal density had similar evolution with the previously established cases, the transverse density distribution was strongly modified by the $s$-dependent strong focusing in the quadrupole beam line. While this modulation is not important for the $\mathrm{CeC}$ process, the obtained results complete the physical picture and provide details of the modulation process in 3D.

Finally, we had performed a large number of simulations for various trajectories and velocities of ions, which allow us to accurately simulate the input into the FEL code we are using for the numerical study of the modulation amplification. In the next step, we will add an experimentally measured electron beam distribution and the simulation of its interaction with ions will be used for predicting the performance of $\mathrm{CeC}$.

\section{ACKNOWLEDGMENTS}

The authors are thankful to Dr. Thomas Roser for his genuine interest and his continuous support of the project. This work was supported by NSF Grant No. PHY1415252 and by Brookhaven Science Associates, LLC under Contract No. DEAC0298CH10886 with the U.S. Department of Energy.

[1] V. N. Litvinenko and Y. S. Derbenev, Free electron lasers and high-energy electron cooling, in Proceedings of the FEL07(29th International Free Electron Laser Conference) (Budker INP, Novosibirsk, 2007); Report No. BNL-795092007-CP.

[2] V. N. Litvinenko, Coherent electron cooling, in Proceedings of the 23rd Particle Accelerator Conference, Vancouver, Canada, 2009 (IEEE, Piscataway, 2009), FR1GRI01.

[3] V. N. Litvinenko and Y. S. Derbenev, Coherent Electron Cooling, Phys. Rev. Lett. 102, 114801 (2009).

[4] V. N. Litvinenko, Y. Jing, I. Pinayev, G. Wang, R. Samulyak, and D. Ratner, Testing aspects of advanced coherent electron cooling, in Proceedings of 6th International Particle Accelerator Conference (IPAC 2015), Richmond, VA, USA, 2015, MOPJE062 (Report No. BNL-108153-2015-CP).

[5] D. Ratner, Microbunched Electron Cooling for High-Energy Hadron Beams, Phys. Rev. Lett. 111, 084802 (2013).

[6] V. N. Litvinenko, G. Wang, G. I. Bell, D. L. Bruhwiler, A. Elizarov, Y. Hao, and Y. Jing, Advances in coherent electron cooling, in Proceedings of COOL 2013 workshop, 
Mürren, Switzerland, 2013, p. 175, ISBN: 978-3-95450140-3.

[7] G. Wang abd M. Blaskiewicz, Dynamics of ion shielding in an anisotropic electron plasma, Phys. Rev. E 78, 026413 (2008).

[8] A. Elizarov and V. Litvinenko, Dynamics of shielding of a moving charged particle in a confined electron plasma, Phys. Rev. ST Accel. Beams 18, 044001 (2015).

[9] G. I. Bell, I. Pogorelov, B. T. Schwartz, D. L. Bruhwiler, V. Litvinenko, G. Wang, and Y. Hao, Modulator simulations for coherent electron cooling using a variable density electron beam, arXiv:1404.2320.

[10] K. Yu and R. Samulyak, Space code for beam-plasma interaction, Proceedings of 6th International Particle Accelerator Conference (IPAC 2015), Richmond, VA, USA, 2015, MOPMN012.

[11] X. Wang, R. Samulyak, J. Jiao, and K. Yu, Adaptive Particle-in-cloud method for optimal solutions to VlasovPoisson equation, J. Comput. Phys. 316, 682 (2016).

[12] K. Yu, R. Samulyak, K. Yonehara, and B. Freemire, Simulation of beam-induced plasma in gas-filled $\mathrm{rf}$ cavities, Phys. Rev. Accel. Beams 20, 032002 (2017).

[13] J. Ma, R. Samulyak, K. Yu, V. N. Litvinenko, and G. Wang, Simulation of beam-induced plasma for the mitigation of beam-beam effects, in Proceedings of 6th International Particle Accelerator Conference (IPAC 2015), Richmond, VA, USA, 2015, MOPMN015 (Report No. BNL-108154-2015-CP).

[14] Y. Jing, V. N. Litvinenko, Y. Hao, and G. Wang, Model independent description of amplification and saturation using Green's function, arXiv:1505.04735.
[15] G. Bell, D. Bruhwiler, B. Schwartz, I. Pogorelov, V. N. Litvinenko, G. Wang, and Y. Hao, Vlasov and PIC simulations of a modulator section for coherent electron cooling, in Proceedings of the 24th Particle Accelerator Conference, PAC-2011, New York, 2011 (IEEE, New York, 2011), MOP067.

[16] G. Wang, V. N. Litvinenko, and M. Blaskiewicz, Energy modulation in coherent electron cooling, in Proceedings of the 4th International Particle Accelerator Conference, IPAC-2013, Shanghai, China, 2013 (CERN, Geneva, 2013), MOPEA083.

[17] B. D. Muratori, J. K. Jones, and A. Wolski, Analytical expressions for fringe fields in multipole magnets, Phys. Rev. ST Accel. Beams 18, 064001 (2015).

[18] L. Deniau, H. Grote, G. Roy, and F. Schmidt, The MAD-X Program (Methodical Accelerator Design), Version 5.04.02, User's Reference Manual, http://madx.web.cern.ch/madx/ webguide/manual.html.

[19] J. Qiang, S. Lidia, R. D. Ryne, and C. Limborg-Deprey, Three-dimensional quasistatic model for high brightness beam dynamics simulation, Phys. Rev. ST Accel. Beams 9 , 044204 (2006).

[20] V. N. Litvinenko and G. Wang, Compensating tune spread induced by space charge in bunched beams, Phys. Rev. ST Accel. Beams 17, 114401 (2014).

[21] J. Ma, Numerical algorithms for Vlasov-Poisson equation and applications to coherent electron cooling, Ph.D. thesis, Stony Brook University, 2017.

[22] G. Wang and V. Litvinenko, Estimation of bunching factor due to Debye shielding (private communication). 